\title{
Search for Higgs boson production in trilepton and like-charge electron-muon final states with the DO detector
}

V. M. Abazov, ${ }^{32}$ B. Abbott,${ }^{67}$ B. S. Acharya,${ }^{26}$ M. Adams,${ }^{46}$ T. Adams,${ }^{44}$ G. D. Alexeev, ${ }^{32}$ G. Alkhazov, ${ }^{36}$ A. Alton, ${ }^{56, *}$ A. Askew, ${ }^{44}$ S. Atkins, ${ }^{54}$ K. Augsten, ${ }^{7}$ C. Avila,${ }^{5}$ F. Badaud,${ }^{10}$ L. Bagby,${ }^{45}$ B. Baldin, ${ }^{45}$ D. V. Bandurin, ${ }^{44}$ S. Banerjee, ${ }^{26}$ E. Barberis, ${ }^{55}$ P. Baringer, ${ }^{53}$ J. F. Bartlett ${ }^{45}$ U. Bassler, ${ }^{15}$ V. Bazterra, ${ }^{46}$ A. Bean,${ }^{53}$ M. Begalli, ${ }^{2}$ L. Bellantoni ${ }^{45}$ S. B. Beri, ${ }^{24}$ G. Bernardi,${ }^{14}$ R. Bernhard,${ }^{19}$ I. Bertram, ${ }^{39}$ M. Besançon, ${ }^{15}$ R. Beuselinck,${ }^{40}$ P. C. Bhat, ${ }^{45}$ S. Bhatia ${ }^{58}$ V. Bhatnagar, ${ }^{24}$ G. Blazey, ${ }^{47}$ S. Blessing, ${ }^{44}$ K. Bloom, ${ }^{59}$ A. Boehnlein,${ }^{45}$ D. Boline,${ }^{64}$ E. E. Boos,${ }^{34}$ G. Borissov, ${ }^{39}$ A. Brandt, ${ }^{70}$ O. Brandt, ${ }^{20}$ R. Brock,${ }^{57}$ A. Bross,${ }^{45}$ D. Brown,${ }^{14}$ X. B. Bu, ${ }^{45}$ M. Buehler, ${ }^{45}$ V. Buescher,${ }^{21}$ V. Bunichev, ${ }^{34}$ S. Burdin, ${ }^{39, \dagger}$ C.P. Buszello, ${ }^{38}$ E. Camacho-Pérez,${ }^{29}$ B. C. K. Casey, ${ }^{45}$ H. Castilla-Valdez, ${ }^{29}$

S. Caughron, ${ }^{57}$ S. Chakrabarti, ${ }^{64}$ D. Chakraborty, ${ }^{47}$ K. M. Chan,${ }^{51}$ A. Chandra, ${ }^{72}$ E. Chapon, ${ }^{15}$ G. Chen, ${ }^{53}$ S. W. Cho, ${ }^{28}$ S. Choi ${ }^{28}$ B. Choudhary, ${ }^{25}$ S. Cihangir, ${ }^{45}$ D. Claes, ${ }^{59}$ J. Clutter, ${ }^{53}$ M. Cooke, ${ }^{45}$ W. E. Cooper, ${ }^{45}$ M. Corcoran, ${ }^{72}$ F. Couderc, ${ }^{15}$ M.-C. Cousinou, ${ }^{12}$ D. Cutts, ${ }^{69}$ A. Das, ${ }^{42}$ G. Davies, ${ }^{40}$ S. J. de Jong, ${ }^{30,31}$ E. De La Cruz-Burelo, ${ }^{29}$ F. Déliot,${ }^{15}$ R. Demina ${ }^{63}$ D. Denisov, ${ }^{45}$ S. P. Denisov, ${ }^{35}$ S. Desai ${ }^{45}$ C. Deterre,${ }^{20,8}$ K. DeVaughan, ${ }^{59}$ H. T. Diehl,${ }^{45}$ M. Diesburg, ${ }^{45}$ P.F. Ding ${ }^{41}$ A. Dominguez, ${ }^{59}$ A. Dubey, ${ }^{25}$ L. V. Dudko, ${ }^{44}$ A. Duperrin, ${ }^{12}$ S. Dutt, ${ }^{24}$ A. Dyshkant,${ }^{47}$ M. Eads, ${ }^{47}$ D. Edmunds, ${ }^{57}$ J. Ellison, ${ }^{43}$ V. D. Elvira ${ }^{45}$ Y. Enari, ${ }^{14}$ H. Evans, ${ }^{49}$ V. N. Evdokimov, ${ }^{35}$ L. Feng,${ }^{47}$

T. Ferbel,${ }^{63}$ F. Fiedler, ${ }^{21}$ F. Filthaut,${ }^{30,31}$ W. Fisher, ${ }^{57}$ H. E. Fisk, ${ }^{45}$ M. Fortner ${ }^{47}$ H. Fox ${ }^{39}$ S. Fuess,${ }^{45}$

A. Garcia-Bellido, ${ }^{63}$ J. A. García-González, ${ }^{29}$ G. A. García-Guerra, ${ }^{29,}{ }^{\ddagger}$ V. Gavrilov, ${ }^{33}$ W. Geng, ${ }^{12,57}$ C. E. Gerber, ${ }^{46}$ Y. Gershtein, ${ }^{60}$ G. Ginther, ${ }^{45,63}$ G. Golovanov, ${ }^{32}$ P. D. Grannis, ${ }^{64}$ S. Greder, ${ }^{16}$ H. Greenlee, ${ }^{45}$ G. Grenier,${ }^{17} \mathrm{Ph}$. Gris, ${ }^{10}$ J.-F. Grivaz, ${ }^{13}$ A. Grohsjean, ${ }^{15}{ }^{\S}$ S. Grünendahl,${ }^{45}$ M. W. Grünewald ${ }^{27}$ T. Guillemin, ${ }^{13}$ G. Gutierrez, ${ }^{45}$ P. Gutierrez,${ }^{67}$ J. Haley, ${ }^{55}$ L. Han, ${ }^{4}$ K. Harder, ${ }^{41}$ A. Harel,${ }^{63}$ J. M. Hauptman, ${ }^{52}$ J. Hays, ${ }^{40}$ T. Head, ${ }^{41}$ T. Hebbeker, ${ }^{18}$ D. Hedin, ${ }^{47}$

H. Hegab, ${ }^{68}$ A. P. Heinson, ${ }^{43}$ U. Heintz ${ }^{69}$ C. Hensel, ${ }^{20}$ I. Heredia-De La Cruz, ${ }^{29}$ K. Herner,${ }^{56}$ G. Hesketh, ${ }^{41, \pi}$ M. D. Hildreth, ${ }^{51}$ R. Hirosky, ${ }^{73}$ T. Hoang ${ }^{44}$ J. D. Hobbs, ${ }^{64}$ B. Hoeneisen, ${ }^{9}$ J. Hogan, ${ }^{72}$ M. Hohlfeld, ${ }^{21}$ I. Howley ${ }^{70}$ Z. Hubacek, ${ }^{7,15}$ V. Hynek, ${ }^{7}$ I. Iashvili, ${ }^{62}$ Y. Ilchenko, ${ }^{71}$ R. Illingworth, ${ }^{45}$ A. S. Ito, ${ }^{45}$ S. Jabeen, ${ }^{69}$ M. Jaffré, ${ }^{13}$ A. Jayasinghe, ${ }^{67}$ M. S. Jeong, ${ }^{28}$ R. Jesik, ${ }^{40}$ P. Jiang, ${ }^{4}$ K. Johns, ${ }^{42}$ E. Johnson,${ }^{57}$ M. Johnson, ${ }^{45}$ A. Jonckheere, ${ }^{45}$ P. Jonsson, ${ }^{40}$ J. Joshi, ${ }^{43}$ A. W. Jung, ${ }^{45}$ A. Juste, ${ }^{37}$ E. Kajfasz, ${ }^{12}$ D. Karmanov, ${ }^{34}$ I. Katsanos, ${ }^{59}$ R. Kehoe, ${ }^{71}$ S. Kermiche, ${ }^{12}$ N. Khalatyan, ${ }^{45}$ A. Khanov, ${ }^{68}$ A. Kharchilava, ${ }^{62}$ Y. N. Kharzheev, ${ }^{32}$ I. Kiselevich, ${ }^{33}$ J. M. Kohli, ${ }^{24}$ A. V. Kozelov, ${ }^{35}$ J. Kraus, ${ }^{58}$ A. Kumar, ${ }^{62}$ A. Kupco, ${ }^{8}$ T. Kurča, ${ }^{17}$ V. A. Kuzmin, ${ }^{34}$ S. Lammers, ${ }^{49}$ P. Lebrun, ${ }^{17}$ H. S. Lee, ${ }^{28}$ S. W. Lee, ${ }^{52}$ W. M. Lee, ${ }^{44}$ X. Lei ${ }^{42}$ J. Lellouch, ${ }^{14}$ D. Li,${ }^{14}$ H. Li ${ }^{73}$ L. Li, ${ }^{43}$ Q. Z. Li, ${ }^{45}$ J. K. Lim, ${ }^{28}$ D. Lincoln, ${ }^{45}$ J. Linnemann, ${ }^{57}$ V. V. Lipaev, ${ }^{35}$ R. Lipton, ${ }^{45}$ H. Liu, ${ }^{71}$ Y. Liu, ${ }^{4}$ A. Lobodenko, ${ }^{36}$ M. Lokajicek ${ }^{8}$ R. Lopes de Sa ${ }^{64}$ R. Luna-Garcia, ${ }^{29, * *}$ A. L. Lyon, ${ }^{45}$ A. K. A. Maciel, ${ }^{1}$ R. Magaña-Villalba, ${ }^{29}$ S. Malik, ${ }^{59}$ V.L. Malyshev, ${ }^{32}$ J. Mansour, ${ }^{20}$ J. Martínez-Ortega,${ }^{29}$ R. McCarthy, ${ }^{64}$ C. L. McGivern, ${ }^{41}$ M. M. Meijer, ${ }^{30,31}$ A. Melnitchouk, ${ }^{45}$ D. Menezes, ${ }^{47}$ P. G. Mercadante, ${ }^{3}$ M. Merkin, ${ }^{34}$ A. Meyer, ${ }^{18}$ J. Meyer, ${ }^{20,8 \S}$ F. Miconi, ${ }^{16}$ N. K. Mondal, ${ }^{26}$ M. Mulhearn, ${ }^{73}$ E. Nagy, ${ }^{12}$ M. Naimuddin, ${ }^{25}$ M. Narain, ${ }^{69}$ R. Nayyar, ${ }^{42}$ H. A. Neal,${ }^{56}$ J. P. Negret, ${ }^{5}$ P. Neustroev, ${ }^{36}$ H. T. Nguyen, ${ }^{73}$ T. Nunnemann, ${ }^{22}$ J. Orduna, ${ }^{72}$ N. Osman, ${ }^{12}$ J. Osta, ${ }^{51}$ M. Padilla, ${ }^{43}$ A. Pal, ${ }^{70}$ N. Parashar, ${ }^{50}$ V. Parihar, ${ }^{69}$ S. K. Park, ${ }^{28}$ R. Partridge,${ }^{69, \|}$ N. Parua, ${ }^{49}$ A. Patwa, ${ }^{65,\|\|}$ B. Penning, ${ }^{45}$ M. Perfilov, ${ }^{34}$ Y. Peters,${ }^{20}$ K. Petridis, ${ }^{41}$ G. Petrillo, ${ }^{63}$ P. Pétroff, ${ }^{13}$ M.-A. Pleier, ${ }^{65}$ P. L. M. Podesta-Lerma, ${ }^{29, \dagger}$ V. M. Podstavkov, ${ }^{45}$ A. V. Popov, ${ }^{35}$ M. Prewitt, ${ }^{72}$ D. Price,${ }^{49}$ N. Prokopenko, ${ }^{35}$ J. Qian, ${ }^{56}$ A. Quadt,${ }^{20}$ B. Quinn, ${ }^{58}$ M. S. Rangel, ${ }^{1}$

P. N. Ratoff, ${ }^{39}$ I. Razumov, ${ }^{35}$ I. Ripp-Baudot, ${ }^{16}$ F. Rizatdinova, ${ }^{68}$ M. Rominsky, ${ }^{45}$ A. Ross, ${ }^{39}$ C. Royon, ${ }^{15}$ P. Rubinov, ${ }^{45}$

R. Ruchti, ${ }^{51}$ G. Sajot, ${ }^{11}$ P. Salcido, ${ }^{47}$ A. Sánchez-Hernández, ${ }^{29}$ M. P. Sanders, ${ }^{22}$ A. S. Santos,,$+\neq$ G. Savage, ${ }^{45}$

L. Sawyer, ${ }^{54}$ T. Scanlon, ${ }^{40}$ R. D. Schamberger,${ }^{64}$ Y. Scheglov, ${ }^{36}$ H. Schellman, ${ }^{48}$ C. Schwanenberger, ${ }^{41}$

R. Schwienhorst, ${ }^{57}$ J. Sekaric, ${ }^{53}$ H. Severini, ${ }^{67}$ E. Shabalina, ${ }^{20}$ V. Shary, ${ }^{15}$ S. Shaw, ${ }^{57}$ A. A. Shchukin, ${ }^{35}$

R. K. Shivpuri, ${ }^{25}$ V. Simak, ${ }^{7}$ P. Skubic,${ }^{67}$ P. Slattery, ${ }^{63}$ D. Smirnov, ${ }^{51}$ K. J. Smith, ${ }^{62}$ G. R. Snow, ${ }^{59}$ J. Snow, ${ }^{66}$ S. Snyder ${ }^{65}$ S. Söldner-Rembold, ${ }^{41}$ L. Sonnenschein, ${ }^{18}$ K. Soustruznik, ${ }^{6}$ J. Stark, ${ }^{11}$ D. A. Stoyanova, ${ }^{35}$ M. Strauss,${ }^{67}$ L. Suter ${ }^{41}$ P. Svoisky, ${ }^{67}$ M. Titov, ${ }^{15}$ V. V. Tokmenin, ${ }^{32}$ Y.-T. Tsai, ${ }^{63}$ D. Tsybychev ${ }^{64}$ B. Tuchming,,${ }^{15}$ C. Tully,${ }^{61}$

L. Uvarov, ${ }^{36}$ S. Uvarov, ${ }^{36}$ S. Uzunyan, ${ }^{47}$ R. Van Kooten, ${ }^{49}$ W. M. van Leeuwen, ${ }^{30}$ N. Varelas, ${ }^{46}$ E. W. Varnes, ${ }^{42}$ I. A. Vasilyev, ${ }^{35}$ A. Y. Verkheev, ${ }^{32}$ L. S. Vertogradov, ${ }^{32}$ M. Verzocchi,${ }^{45}$ M. Vesterinen, ${ }^{41}$ D. Vilanova, ${ }^{15}$ P. Vokac, ${ }^{7}$

H. D. Wahl, ${ }^{44}$ M. H. L. S. Wang, ${ }^{45}$ J. Warchol, ${ }^{51}$ G. Watts ${ }^{74}$ M. Wayne, ${ }^{51}$ J. Weichert, ${ }^{21}$ L. Welty-Rieger, ${ }^{48}$

A. White, ${ }^{70}$ D. Wicke, ${ }^{23}$ M. R. J. Williams, ${ }^{39}$ G. W. Wilson, ${ }^{53}$ M. Wobisch,${ }^{54}$ D. R. Wood,${ }^{55}$ T. R. Wyatt,${ }^{41}$ Y. Xie, ${ }^{45}$ R. Yamada, ${ }^{45}$ S. Yang, ${ }^{4}$ T. Yasuda ${ }^{45}$ Y. A. Yatsunenko, ${ }^{32}$ W. Ye ${ }^{64}$ Z. Ye, ${ }^{45}$ H. Yin, ${ }^{45}$ K. Yip, ${ }^{65}$ S. W. Youn, ${ }^{45}$ J. M. Yu, ${ }^{56}$ J. Zennamo, ${ }^{62}$ T. G. Zhao, ${ }^{41}$ B. Zhou, ${ }^{56}$ J. Zhu, ${ }^{56}$ M. Zielinski, ${ }^{63}$ D. Zieminska, ${ }^{49}$ and L. Zivkovic ${ }^{14}$ 


\section{(D0 Collaboration)}

${ }^{1}$ LAFEX, Centro Brasileiro de Pesquisas Físicas, Rio de Janeiro, Brazil

${ }^{2}$ Universidade do Estado do Rio de Janeiro, Rio de Janeiro, Brazil

${ }^{3}$ Universidade Federal do ABC, Santo André, Brazil

${ }^{4}$ University of Science and Technology of China, Hefei, People's Republic of China

${ }^{5}$ Universidad de los Andes, Bogotá, Colombia

${ }^{6}$ Charles University, Faculty of Mathematics and Physics, Center for Particle Physics, Prague, Czech Republic

${ }^{7}$ Czech Technical University in Prague, Prague, Czech Republic

${ }^{8}$ Center for Particle Physics, Institute of Physics, Academy of Sciences of the Czech Republic, Prague, Czech Republic

${ }^{9}$ Universidad San Francisco de Quito, Quito, Ecuador

${ }^{10}$ LPC, Université Blaise Pascal, CNRS/IN2P3, Clermont, France

${ }^{11}$ LPSC, Université Joseph Fourier Grenoble 1, CNRS/IN2P3, Institut National Polytechnique de Grenoble, Grenoble, France

${ }^{12}$ CPPM, Aix-Marseille Université, CNRS/IN2P3, Marseille, France

${ }^{13}$ LAL, Université Paris-Sud, CNRS/IN2P3, Orsay, France

${ }^{14}$ LPNHE, Universités Paris VI and VII, CNRS/IN2P3, Paris, France

${ }^{15}$ CEA, Irfu, SPP, Saclay, France

${ }^{16} I P H C$, Université de Strasbourg, CNRS/IN2P3, Strasbourg, France

${ }^{17}$ IPNL, Université Lyon 1, CNRS/IN2P3, Villeurbanne, France and Université de Lyon, Lyon, France

${ }^{18}$ III. Physikalisches Institut A, RWTH Aachen University, Aachen, Germany

${ }^{19}$ Physikalisches Institut, Universität Freiburg, Freiburg, Germany

${ }^{20}$ II. Physikalisches Institut, Georg-August-Universität Göttingen, Göttingen, Germany

${ }^{21}$ Institut für Physik, Universität Mainz, Mainz, Germany

${ }^{22}$ Ludwig-Maximilians-Universität München, München, Germany

${ }^{23}$ Fachbereich Physik, Bergische Universität Wuppertal, Wuppertal, Germany

${ }^{24}$ Panjab University, Chandigarh, India

${ }^{25}$ Delhi University, Delhi, India

${ }^{26}$ Tata Institute of Fundamental Research, Mumbai, India

${ }^{27}$ University College Dublin, Dublin, Ireland

${ }^{28}$ Korea Detector Laboratory, Korea University, Seoul, Korea

${ }^{29}$ CINVESTAV, Mexico City, Mexico

${ }^{30}$ Nikhef, Science Park, Amsterdam, The Netherlands

${ }^{31}$ Radboud University Nijmegen, Nijmegen, The Netherlands

${ }^{32}$ Joint Institute for Nuclear Research, Dubna, Russia

${ }^{33}$ Institute for Theoretical and Experimental Physics, Moscow, Russia

${ }^{34}$ Moscow State University, Moscow, Russia

${ }^{35}$ Institute for High Energy Physics, Protvino, Russia

${ }^{36}$ Petersburg Nuclear Physics Institute, St. Petersburg, Russia

${ }^{37}$ Institució Catalana de Recerca i Estudis Avançats (ICREA) and Institut de Física d'Altes Energies (IFAE), Barcelona, Spain

${ }^{38}$ Uppsala University, Uppsala, Sweden

${ }^{39}$ Lancaster University, Lancaster LA1 4YB, United Kingdom

${ }^{40}$ Imperial College London, London SW7 2AZ, United Kingdom

${ }^{41}$ The University of Manchester, Manchester M13 9PL, United Kingdom

${ }^{42}$ University of Arizona, Tucson, Arizona 85721, USA

${ }^{43}$ University of California Riverside, Riverside, California 92521, USA

${ }^{44}$ Florida State University, Tallahassee, Florida 32306, USA

${ }^{45}$ Fermi National Accelerator Laboratory, Batavia, Illinois 60510, USA

${ }^{46}$ University of Illinois at Chicago, Chicago, Illinois 60607, USA

${ }^{47}$ Northern Illinois University, DeKalb, Illinois 60115, USA

${ }^{48}$ Northwestern University, Evanston, Illinois 60208, USA

${ }^{49}$ Indiana University, Bloomington, Indiana 47405, USA

${ }^{50}$ Purdue University Calumet, Hammond, Indiana 46323, USA

${ }^{51}$ University of Notre Dame, Notre Dame, Indiana 46556, USA

${ }^{52}$ Iowa State University, Ames, Iowa 50011, USA

${ }^{53}$ University of Kansas, Lawrence, Kansas 66045, USA

${ }^{54}$ Louisiana Tech University, Ruston, Louisiana 71272, USA

${ }^{55}$ Northeastern University, Boston, Massachusetts 02115, USA

${ }^{56}$ University of Michigan, Ann Arbor, Michigan 48109, USA

${ }^{57}$ Michigan State University, East Lansing, Michigan 48824, USA 


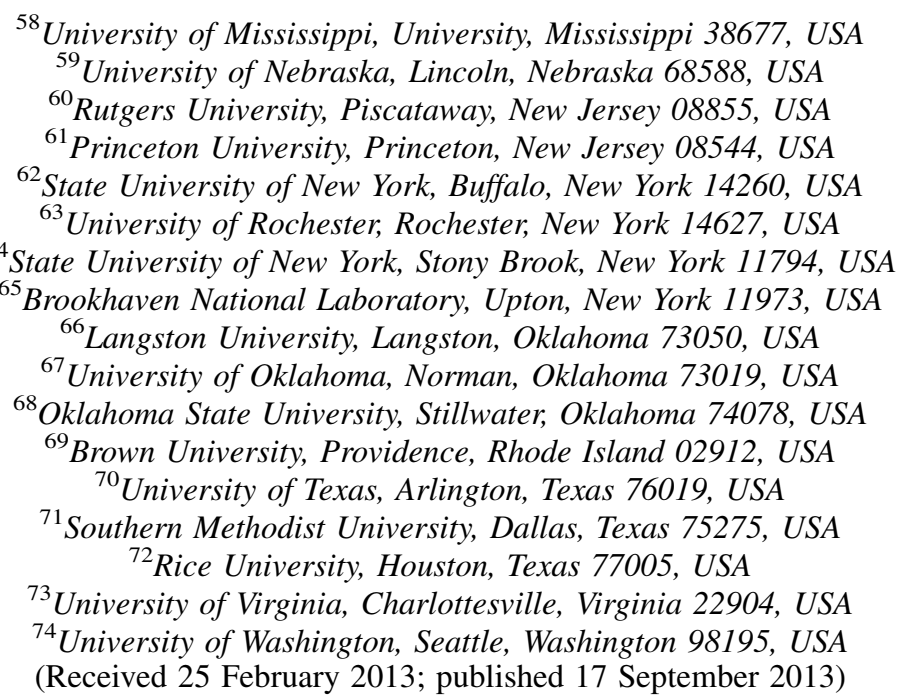

We present a search for Higgs bosons in multilepton final states in $p \bar{p}$ collisions at $\sqrt{s}=1.96 \mathrm{TeV}$ recorded with the D0 detector at the Fermilab Tevatron Collider, using the full Run II data set with integrated luminosities of up to $9.7 \mathrm{fb}^{-1}$. The multilepton states considered are ee $\mu, e \mu \mu, \mu \tau_{h} \tau_{h}$, and like-charge $e^{ \pm} \mu^{ \pm}$pairs. These channels directly probe the $H V V(V=W, Z)$ coupling of the Higgs boson in production and decay. The $\mu \tau_{h} \tau_{h}$ channel is also sensitive to $H \rightarrow \tau^{+} \tau^{-}$decays. Upper limits at the 95\% C.L on the rate of standard model Higgs boson production are derived in the mass range $100 \leq$ $M_{H} \leq 200 \mathrm{GeV}$. The expected and observed limits are a factor of 6.3 and 8.4 above the predicted standard model cross section at $M_{H}=125 \mathrm{GeV}$. We also interpret the data in a fermiophobic Higgs boson model.

DOI: 10.1103/PhysRevD.88.052009

PACS numbers: $14.80 . \mathrm{Ec}, 14.80 . \mathrm{Bn}$

\section{INTRODUCTION}

The Higgs boson is predicted by the standard model $(\mathrm{SM})$ as a consequence of the breaking of the electroweak symmetry, which gives mass to the weak gauge bosons. The ATLAS and CMS Collaborations at the CERN Large Hadron Collider have recently reported the observation of a Higgs-like boson at a mass of $M_{H} \approx 125 \mathrm{GeV}$, primarily in $\gamma \gamma$ and $Z Z$ final states [1,2]. Combining searches in the channel where the Higgs boson is produced in association with a $W$ or $Z$ boson, the CDF and D0 Collaborations have found evidence for Higgs boson decay into $b \bar{b}$ pairs [3].

\footnotetext{
*Visitor from Augustana College, Sioux Falls, SD, USA.

Visitor from The University of Liverpool, Liverpool, UK.

${ }^{*}$ Visitor from UPIITA-IPN, Mexico City, Mexico.

${ }^{\S}$ Visitor from DESY, Hamburg, Germany.

"Visitor from SLAC, Menlo Park, CA, USA.

"Visitor from University College London, London, UK.

**Visitor from Centro de Investigacion en Computacion - IPN, Mexico City, Mexico.

${ }^{\dagger \dagger}$ Visitor from ECFM, Universidad Autonoma de Sinaloa, Culiacán, Mexico.

${ }^{\ddagger}$ Visitor from Universidade Estadual Paulista, São Paulo, Brazil.

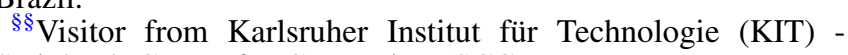
Steinbuch Centre for Computing (SCC).

${ }^{\|\|}$Visitor from Office of Science, U.S. Department of Energy, Washington, D.C. 20585, USA.
}

In this article, we study final states with multiple charged leptons, including electrons, muons, and hadronically decaying tau leptons $\left(\tau_{h}\right)$. We present the first Higgs boson search performed in the trilepton final states $e e \mu$, $e \mu \mu$, and $\mu \tau_{h} \tau_{h}$ with the $\mathrm{D} 0$ detector.

We also consider the production of like-charge $e^{ \pm} \mu^{ \pm}$ pairs. This final state has the advantage of reduced background from $Z$ boson decay that is present in opposite-charge $e^{+} e^{-}, \mu^{+} \mu^{-}$, and $e^{ \pm} \mu^{\mp}$ final states [4]. This analysis supersedes the previous searches in $e^{ \pm} \mu^{ \pm}$final states, which used integrated luminosities of up to $5.3 \mathrm{fb}^{-1}$ [5].

The main Higgs boson production mechanisms relevant for this analysis are associated $W H$ and $Z H$ production and gluon-gluon fusion. The contribution from vector boson fusion is small and therefore neglected. The multilepton channels are sensitive to Higgs boson decays into $W^{+} W^{-}$ and $Z Z$ pairs, where the vector bosons $(V)$ decay leptonically. These channels therefore directly probe the $H V V$ coupling in production and decay. The trilepton searches are also sensitive to $H \rightarrow \tau^{+} \tau^{-}$decays from associated production $(W H, Z H)$ through hadronic tau decays in the $\mu \tau_{h} \tau_{h}$ channel and through leptonic tau decays in the $e e \mu$ and $e \mu \mu$ channels. Searches for $H \rightarrow \tau^{+} \tau^{-}$decays in final states with additional jets have also been performed using the full Run II data set [6].

We also interpret the data in a fermiophobic model with a Higgs boson that does not couple to fermions but 
couples to $W$ and $Z$ bosons with SM strengths. Such searches have been conducted at the CERN $e^{+} e^{-}$ Collider LEP [7-10] and at the Fermilab Tevatron Collider [11,12]. The CMS Collaboration excludes fermiophobic Higgs bosons with $M_{H}<124.5 \mathrm{GeV}, 127<$ $M_{H}<147.5 \mathrm{GeV}$, and $155<M_{H}<180 \mathrm{GeV}$ at the 95\% C.L. in a model that assumes the couplings of the Higgs boson to other bosons are SM-like [13].

Most results in this article are based on the full Run II data set collected with the D0 detector at the Fermilab Tevatron and correspond to an integrated luminosity of $9.7 \mathrm{fb}^{-1}$. The analysis of the $\mu \tau_{h} \tau_{h}$ final state only uses data recorded after June 2006 with an integrated luminosity of $8.6 \mathrm{fb}^{-1}$. The results provide an important input for the combined Higgs boson search performed by the D0 Collaboration [14] and for the Tevatron combination [15].

\section{DO DETECTOR}

The D0 detector [16] comprises tracking detectors and calorimeters. Silicon microstrip detectors and a scintillating fiber tracker are used to reconstruct charged particle tracks within a $2 \mathrm{~T}$ solenoid. A liquid-argon and uranium calorimeter has a central section (CC) covering pseudorapidities [17] $\left|\eta_{d}\right|$ up to $\approx 1.1$, and two end calorimeters $(\mathrm{EC})$ that extend coverage to $\left|\eta_{d}\right| \approx 4.2$. The calorimeters consist of electromagnetic (EM) and hadronic sections segmented longitudinally in several layers. Muons are identified by combining tracks with patterns of hits in the muon spectrometer, which lies outside the calorimeter and consists of a layer of tracking detectors and scintillation trigger counters in front of a $1.8 \mathrm{~T}$ toroid, followed by two similar layers after the toroid [18]. Trigger decisions are based on partial information from the tracking detectors, calorimeters, and muon spectrometer [16].

\section{EVENT SIMULATION}

All background processes are simulated using Monte Carlo (MC) event generators, except the $Z \gamma$ background in the $e \mu \mu$ channel and the multijet background, which are determined from data. The $W+$ jets, $Z / \gamma^{*} \rightarrow$ $\ell^{+} \ell^{-}+$jet, and $t \bar{t}$ processes are generated using ALPGEN [19] with showering and hadronization provided by PYTHIA [20]. Diboson production ( $W W, W Z$, and $Z Z)$ and signal events are simulated using PYTHIA. All these simulations use the CTEQ6L1 [21] parton distribution functions (PDFs). Associated production of Higgs bosons ( $W H$ and $\mathrm{ZH}$ ) and gluon-gluon fusion are generated in $5 \mathrm{GeV}$ increments of $M_{H}$ in the range $100 \leq M_{H} \leq 200 \mathrm{GeV}$. Tau lepton decays are simulated with TAUOLA [22], which includes a full treatment of the tau polarization.

Next-to-leading order (NLO) quantum chromodynamics calculations of cross sections are used to normalize the background contribution of $t \bar{t}$ [23] and diboson [24] processes. The $W Z$ production cross section is corrected for $W \gamma^{*}$ interference using POWHEG [25]. The cross section for $W / Z+$ jets production is normalized to a next-to-NLO (NNLO) calculation [26]. The transverse momentum $\left(p_{T}\right)$ spectrum of $Z$ bosons, defined as the momentum component transverse to the beam direction, is corrected to match the measured distributions [27]. The correction factor for the $p_{T}$ spectrum of $W$ bosons is the product of the $Z$ boson correction factor and the ratio of the $p_{T}$ spectra of $W$ and $Z$ bosons calculated at NNLO [28].

The cross sections for $\mathrm{VH}$ associated production are calculated at NNLO [29,30]. The NNLO calculation of Higgs boson production in gluon-gluon fusion takes into account resummation of soft gluons to next-to-next-toleading-log (NNLL) [31] accuracy. Higher order corrections to the Higgs boson production cross sections are computed with the MSTW 2008 PDF set [32]. The simulated $p_{T}$ spectrum of Higgs bosons from gluon-gluon fusion is corrected using the NNLO and NNLL calculation of HQT [33]. Branching fractions of the Higgs boson decays are calculated using HDECAY [34].

All MC samples are processed through a GEANT [35] simulation of the detector. Data from random beam crossings are overlaid on the $\mathrm{MC}$ events to account for detector noise and additional $p \bar{p}$ interactions. The simulated distributions are corrected for differences between data and simulation in the reconstruction efficiencies and in the distribution of the longitudinal coordinate of the interaction point.

To maximize signal acceptance, we use all events that pass our event selection without requiring a specific trigger condition. The residual efficiency loss from events that are not recorded depends on the event kinematics. We study ratios of kinematic distributions using the inclusive trigger requirements and using a set of specific single lepton triggers. The trigger efficiencies for single leptons have been measured with $Z \rightarrow \ell^{+} \ell^{-}$ decays in data using a tag-and-probe method [36]. For the $e \mu \mu$ and $\mu \tau_{h} \tau_{h}$ final states the measured singlelepton trigger efficiency is scaled by the ratio of the acceptances of the inclusive and the single-lepton trigger conditions determined using data. This factor is applied to kinematic distributions of MC events to account for biases introduced by the inclusive trigger requirement. No correction is applied for the $e e \mu$ and $e^{ \pm} \mu^{ \pm}$final states where the efficiency of the inclusive trigger method is close to $100 \%$.

\section{OBJECT IDENTIFICATION}

The signal comprises electrons, muons, and tau leptons that are isolated from other particles in the detector.

Electrons are identified by requiring electron clusters in the EM calorimeter that are matched with a track in the central 
tracker. The energy is measured in the EM and the first hadronic layers of the calorimeter within a cone of radius $\mathcal{R}=\sqrt{(\Delta \eta)^{2}+(\Delta \phi)^{2}}=0.2$, where $\phi$ is the azimuthal angle. The electron cluster must satisfy a set of criteria: (i) calorimeter isolation fraction, $f_{\text {iso }}=\left(E_{\mathrm{tot}}-E_{\mathrm{EM}}\right) / E_{\mathrm{EM}}$, less than 0.15 for the $\mathrm{CC}$ region and less than 0.1 for EC, where $E_{\text {tot }}$ is the total energy in the cone of radius $\mathcal{R}=0.4$ and $E_{\mathrm{EM}}$ is the EM energy in a cone of radius $\mathcal{R}=0.2$; (ii) fraction of the EM energy to the total energy greater than 0.9 ; and (iii) ratio of the electron's transverse momentum measured by the calorimeter and by the tracking detector, respectively, less than 8 (CC only). In addition, the value of an eight-variable likelihood for electron candidates is required to be $\mathcal{L}_{e}>0.05$ [5]. The electrons must also satisfy a requirement on a neural network discriminant with seven input variables in the $\mathrm{CC}$ and three in the $\mathrm{EC}$ region, including isolation and shower shape variables to improve the discrimination between jets and electrons. The sum of the charged particle tracks' $p_{T}$ in an annulus of $0.05<\mathcal{R}<0.4$ around the electron direction must be less than $3.5 \mathrm{GeV}$ in the $\mathrm{CC}$ and less than $\left(-2.5 \times\left|\eta_{d}\right|+7\right) \mathrm{GeV}$ in the EC. This criterion was optimized by maximizing the efficiency for electrons while suppressing background from jets.

Muons are identified by the presence of at least one track segment reconstructed in the muon spectrometer which is spatially consistent with a track in the central detector, where the momentum and charge are measured by the curvature of this track. Muon isolation is imposed with two isolation variables defined as the scalar sums of the transverse energy in the calorimeter in an annulus of radius $0.1<\mathcal{R}<0.4$ around the muon direction and of the momenta of charged particle tracks within $\mathcal{R}=0.5$. Both variables, divided by the muon $p_{T}$, must be less than 0.2 . To reduce the effects of charge misreconstruction, additional selection criteria on the track quality are applied in the $e^{ \pm} \mu^{ \pm}$channel.

Three types of tau lepton decays into hadrons are identified by their signatures. Type- 1 tau candidates consist of a single track and its associated energy deposit in the calorimeter, without any additional separate energy deposits in the EM section. This signature corresponds mainly to $\tau^{ \pm} \rightarrow \pi^{ \pm} \nu$ decays and also includes leptonic $\tau^{ \pm} \rightarrow e^{ \pm} \nu \nu$ decays. For type- 2 tau candidates, we require a track and its associated calorimeter energy deposit, plus a separate energy deposit in the EM calorimeter consistent with a $\pi^{0} \rightarrow \gamma \gamma$ decay, as expected for $\tau^{ \pm} \rightarrow \pi^{ \pm} \pi^{0} \nu$ decays. Finally, type-3 tau candidates consist of two or three tracks, combined with an energy deposit in the calorimeter. This corresponds mainly to the decays $\tau^{ \pm} \rightarrow \pi^{ \pm} \pi^{ \pm} \pi^{\mp}\left(\pi^{0}\right) \nu$. In this analysis, type- 3 tau candidates are required to have three tracks and an associated net charge of \pm 1 . For each tau type, a neural network is designed to discriminate $\tau_{h}$ from jets. The neural network discriminants are required to be $N N_{\tau}>0.75$ for types 1 and 2 , and $N N_{\tau}>0.95$ for type 3 [37]. The input variables for these neural networks are based on isolation variables for objects and on the spatial distributions of showers. The transverse momentum of the $\tau_{h}$ candidates is calculated using the energy measured in the calorimeter, except for type- $1 \tau_{h}$ candidates where the track information is used if the $p_{T}$ measured in the calorimeter is less than $70 \mathrm{GeV}$.

Variables that include information on the imbalance in transverse energy $\left(\mathscr{E}_{T}\right)$ caused by neutrinos are used to improve the discrimination between signal and background. The $\mathscr{E}_{T}$ is calculated using the transverse energy measured in the calorimeter, corrected for the presence of identified muons. Two modified $\not{E}_{T}$ variables, $\hat{\mathscr{E}}_{T}$ and $\mathcal{S}\left(\mathscr{E}_{T}\right)$, are used to reject events where the $\mathscr{E}_{T}$ arises from detector effects and not from neutrinos. In events where the opening angle $\phi$ between the $\mathscr{E}_{T}$ direction and the nearest lepton or jet is small, the resolution of the $\mathbb{E}_{T}$ measurement is dominated by the uncertainty on the measured lepton or jet energy. Less significance is assigned to this region by using $\hat{\mathbb{E}}_{T}$, defined as $\hat{\mathscr{E}}_{T}=\not \mathbb{E}_{T} \sin \phi$ if $\phi \leq \pi / 2$ and $\hat{\mathscr{E}}_{T}=\not_{T}$ elsewhere. The significance $\mathcal{S}\left(\boldsymbol{E}_{T}\right)$ [38] is defined so that larger values of $\mathcal{S}\left(\mathbb{E}_{T}\right)$ correspond to $\mathbb{E}_{T}$ measurements that are less likely to be caused by fluctuations in jet energies.

Jet variables are used to discriminate between signal and background in the $e \mu \mu$ and $e^{ \pm} \mu^{ \pm}$channels but not in the event selection. We identify jets using a midpoint cone algorithm [39] with a cone size of $\mathcal{R}=0.5$, based on energy deposits in the calorimeter. We correct the energy scale of jets in both data and MC [40]. We require $p_{T}^{\text {jet }}>$ $15 \mathrm{GeV}$ in the $e \mu \mu$ channel and $p_{T}^{\mathrm{jet}}>20 \mathrm{GeV}$ in the $e^{ \pm} \mu^{ \pm}$channel. In both cases the jets must lie within $\left|\eta_{d}^{\mathrm{jet}}\right|<2.4$.

\section{EVENT SELECTION}

The event selection is designed to maximize sensitivity to a SM Higgs boson signal in each channel separately. The leading muon $\left(\mu_{1}\right)$ in the $e \mu \mu$ and $\mu \tau_{h} \tau_{h}$ channels, the leading electron $\left(e_{1}\right)$ in the $e e \mu$ channel, and the electron in the $e^{ \pm} \mu^{ \pm}$channel are required to have $p_{T}>15 \mathrm{GeV}$. All other selected leptons must have a transverse momentum of $p_{T}>10 \mathrm{GeV}$. The pseudorapidity of at least one of the selected muons in all channels except $e e \mu$ and of both $\tau_{h}$ candidates in the $\mu \tau_{h} \tau_{h}$ channel must be $\left|\eta^{\mu}\right|<1.6$ and $\left|\eta^{\tau}\right|<1.5$, respectively. The transverse momentum of type- 1 and type- $2 \tau_{h}$ candidates must be $p_{T}>12.5 \mathrm{GeV}$, and we require $p_{T}>15 \mathrm{GeV}$ for type- $3 \tau_{h}$ candidates.

The leptons in the events originate from a $p \bar{p}$ interaction vertex, which is required to have a longitudinal coordinate located within $60 \mathrm{~cm}$ of the nominal center of the detector. The maximum difference between the longitudinal coordinate at the distance of closest approach to the beam axis for all lepton pairings in an event must be less than $3 \mathrm{~cm}$.

To facilitate combining channels, we ensure that there is no overlap between them. All events with at least two electrons and at least two muons $(e е \mu \mu)$ are included in 
TABLE I. Numbers of events in data, predicted background, and expected signal for $M_{H}=125 \mathrm{GeV}$ after the event selection. The numbers are shown for the different samples separately, together with their total (statistical and systematic) uncertainties.

\begin{tabular}{lcccccc}
\hline \hline & $e e \mu$ & $e \mu \mu_{\mathrm{A}}$ & $e \mu \mu_{\mathrm{B}}$ & $e \mu \mu_{\mathrm{C}}$ & $\mu \tau_{h} \tau_{h}$ & $e^{ \pm} \mu^{ \pm}$ \\
\hline Signal & & & & & & \\
$W H$ & 0.39 & 0.23 & 0.07 & 0.02 & 0.55 & 1.93 \\
$Z H$ & 0.45 & 0.06 & 0.19 & 0.11 & 0.17 & 0.32 \\
$g g \rightarrow H \rightarrow Z Z$ & 0.05 & $<0.01$ & 0.01 & 0.02 & $<0.01$ & $<0.01$ \\
Signal Sum & 0.89 & 0.29 & 0.27 & 0.15 & 0.72 & 2.25 \\
Background & & & & & & \\
$Z \rightarrow e^{+} e^{-}$ & $39.1 \pm 12.8$ & $<0.1$ & $<0.1$ & $<0.1$ & $0.3 \pm 0.1$ & $15.9 \pm 2.4$ \\
$Z \rightarrow \mu^{+} \mu^{-}$ & $<0.1$ & $2.6 \pm 0.9$ & $8.4 \pm 2.9$ & $32.3 \pm 10.6$ & $4.4 \pm 0.6$ & $58.5 \pm 15.2$ \\
$Z \rightarrow \tau^{+} \tau^{-}$ & $1.5 \pm 0.6$ & $0.1 \pm 0.1$ & $0.1 \pm 0.1$ & $<0.1$ & $5.6 \pm 0.7$ & $22.0 \pm 6.8$ \\
$Z \gamma$ & $<0.1$ & $11.8 \pm 1.1$ & $24.2 \pm 2.0$ & $76.9 \pm 5.9$ & $<0.1$ & $<0.1$ \\
Diboson & $37.1 \pm 4.3$ & $3.9 \pm 0.5$ & $19.4 \pm 2.5$ & $9.4 \pm 1.2$ & $9.0 \pm 1.3$ & $36.2 \pm 3.6$ \\
$t \bar{t}$ & $1.2 \pm 0.2$ & $0.5 \pm 0.1$ & $0.3 \pm 0.1$ & $0.1 \pm 0.1$ & $1.4 \pm 0.2$ & $4.1 \pm 2.1$ \\
$W+$ jets & $0.2 \pm 0.1$ & $<0.1$ & $<0.1$ & $<0.1$ & $5.4 \pm 0.7$ & $238.3 \pm 19.0$ \\
Multijet & $<0.1$ & $<0.1$ & $<0.1$ & $<0.1$ & $<0.1$ & $434.5 \pm 87.0$ \\
Background & & & & & & \\
Sum & $79 \pm 15$ & $19 \pm 2$ & $52 \pm 5$ & $119 \pm 11$ & $26 \pm 4$ & $809 \pm 93$ \\
Data & 77 & 16 & 57 & 119 & 22 & 822 \\
\hline \hline
\end{tabular}

the $e e \mu$ sample and removed from the $e \mu \mu$ sample. All events included in the other trilepton final states are removed from the $\mu \tau_{h} \tau_{h}$ sample. We also reject events with an additional electron or muon in the $e^{ \pm} \mu^{ \pm}$channel.

We construct a variable $M\left(\ell \ell \ell \mathscr{E}_{T}\right)$ that is the invariant mass of the three leptons and the $\mathscr{E}_{T}$, where the $\mathscr{E}_{T}$ vector is assumed to have a longitudinal momentum component equal to zero. We require $M\left(\ell \ell \ell \mathbb{E}_{T}\right)>100 \mathrm{GeV}$ for the $e e \mu$ and $e \mu \mu$ final states to reject $Z+$ jets background. To remove $Z \rightarrow \mu \mu$ events with final state radiation, we require $e \mu \mu$ events in the range $75<M(e \mu \mu)<$ $105 \mathrm{GeV}$ to have $\mathscr{E}_{T}>20 \mathrm{GeV}$. In the $\mu \tau_{h} \tau_{h}$ final state, we require $\not E_{T}>15 \mathrm{GeV}$ to reject multijet events and a transverse mass $M_{T}(\mu)>20 \mathrm{GeV}$ to reject $Z \rightarrow \tau \tau$ background. The transverse mass

$$
M_{T}(\ell)=\sqrt{\left.2 p_{T}^{\ell} \dot{E}_{T}(1-\cos \phi)\right)}
$$

is calculated using the azimuthal angle $\phi$ between the charged lepton $(\ell=e, \mu)$ and the direction of the $\mathscr{E}_{T}$.

Muons and electrons must be separated from jets by $\mathcal{R}>0.1$ in the $e \mu \mu$, ee $\mu$, and $e^{ \pm} \mu^{ \pm}$channels. All selected leptons are required to be separated by $\mathcal{R}>0.3$ from each other. This is increased to $\mathcal{R}>0.5$ for the pairings of $\tau_{h}$ candidates and for the pairing between $\tau_{h}$ candidates and muons. The sum of the charges in the $\mu \tau_{h} \tau_{h}$ final state must be \pm 1 . The electron and muon are required to have the same charge in the $e^{ \pm} \mu^{ \pm}$final state. No lepton charge requirements are applied for the $e e \mu$ or $e \mu \mu$ final states to maximize the sensitivity to signal.

We divide the $e \mu \mu$ channel into three samples with different signal and background composition to increase sensitivity to a Higgs boson signal. The $e \mu \mu_{\mathrm{A}}$ sample contains events where the dimuon mass is outside the range $60<M(\mu \mu)<130 \mathrm{GeV}$ and all events with like-charge muons. The second sample $\left(e \mu \mu_{\mathrm{B}}\right)$ contains events with oppositely charged muons, $60<M(\mu \mu)<130 \mathrm{GeV}$, and $\mathcal{S}\left(\not \mathbb{E}_{T}\right)>2$. The $e \mu \mu_{\mathrm{C}}$ sample consists of all remaining events with $60<M(\mu \mu)<130 \mathrm{GeV}$ and $\mathcal{S}\left(\mathbb{E}_{T}\right) \leq 2$.

An overall normalization factor for the $e^{ \pm} \mu^{ \pm}$signal and background MC samples is obtained from the ratio of the number of data to MC events in an opposite-charge $e \mu$ sample in the range $25<M(e \mu)<75 \mathrm{GeV}$, which is dominated by $Z+$ jets events.

The number of events in data, the expected background and signal, after all selection criteria described in this section have been applied, are given in Table I.

\section{INSTRUMENTAL BACKGROUNDS}

Instrumental backgrounds are caused by leptons produced inside jets, low-multiplicity jets that are reconstructed as $\tau_{h}$ candidates, photons or jets misidentified as electrons, and by opposite-charge $e \mu$ pairs where one of the charges is incorrectly measured.

The $W+$ jets background in the $e \mu \mu$ and $e e \mu$ samples is expected to be small, and its contribution is therefore described only by the simulation using the theoretical cross section. Since the $W+$ jets background is expected to contribute more for the $\mu \tau_{h} \tau_{h}$ and $e^{ \pm} \mu^{ \pm}$final states, their normalization is obtained using data and then applied to the simulated kinematic distributions of the $W+$ jets events.

To model the $W+$ jets background for $\mu \tau_{h} \tau_{h}$ final states, we select a data sample enriched in $W+$ jets events. We require that events pass all selection criteria, except the requirements on the $N N_{\tau}$ outputs. In addition, we require 
TABLE II. Set of variables used in training of the BDT for each final state. The charges of the leptons are $q_{\ell}(\ell=e, \mu)$. The angle $\phi\left(\ell_{1}, \ell_{2}\right)$ is taken between the two leptons, and the angle $\phi\left(\ell \ell, \ell^{\prime}\right)$ between the dilepton system $(\ell \ell)$ and the lepton with the different flavor $\left(\ell^{\prime}\right)$. The variables $\mathcal{R}(\ell, \ell)$ and $M_{T 2}\left(\ell \ell^{\prime} \mathbb{E}_{T}\right)$ are calculated for all lepton pairings. The pairings with the smallest and largest values are denoted by $\min \{\}$ and $\max \{\}$, respectively, and $\operatorname{mid}\{\}$ corresponds to the third pairing. The mass, transverse momentum, and pseudorapidity of the trilepton system are denoted by $M(\ell \ell \ell), p_{T}^{\ell \ell \ell}$, and $\eta^{\ell \ell \ell}$, respectively, and $\sum p_{T}^{\ell}$ is the scalar sum of the transverse momenta of the three leptons. The variable $f_{c p}$ is the fraction of charged particle tracks associated with the jet that point back to the same vertex as the leptons. If no jets are present, the jet variables are set to zero. All variables given for the $\mu \tau_{h} \tau_{h}$ channel are used in the first BDT, whereas only $p_{T}^{\tau_{1}}, M(\tau \tau), M_{T}(\mu)$, and $\not E_{T}$ are used in the second BDT. All variables given for the $e^{ \pm} \mu^{ \pm}$channel are used in both BDTs, except $p_{T}^{\text {jet }}$ and $\phi\left(\right.$ jet, $\left.\mathscr{E}_{T}\right)$, which are used in the second BDT only.

\begin{tabular}{|c|c|c|c|c|c|c|c|}
\hline \multirow[b]{2}{*}{$e e \mu$} & \multirow[b]{2}{*}{$e \mu \mu_{\mathrm{A}}$} & \multirow[b]{2}{*}{$e \mu \mu_{\mathrm{B}}$} & \multirow[b]{2}{*}{$e \mu \mu_{\mathrm{C}}$} & \multicolumn{2}{|l|}{$\mu \tau_{h} \tau_{h}$} & \multicolumn{2}{|l|}{$e^{ \pm} \mu^{ \pm}$} \\
\hline & & & & BDT1 & BDT2 & BDT1 & BDT2 \\
\hline \multirow[t]{2}{*}{$\begin{array}{l}p_{T}^{e_{1}}, p_{T}^{e_{2}}, p_{T}^{\mu} \\
p_{T}^{e e}, p_{T}^{e e \mu}\end{array}$} & $\begin{array}{c}p_{T}^{e} \\
p_{T}^{\mu \mu}\end{array}$ & $\begin{array}{c}p_{T}^{e} \\
p_{T}^{\mu \mu}\end{array}$ & $\begin{array}{c}p_{T}^{\mu_{1}}, p_{T}^{e} \\
p_{T}^{e \mu \mu}\end{array}$ & $p_{T}^{\tau_{1}}, p_{T}^{\tau_{2}}, p_{T}^{\mu}$ & $p_{T}^{\tau_{1}}$ & $\begin{array}{c}\mathcal{L}_{e} \\
p_{T}^{e}, p_{T}^{\mu}\end{array}$ & \\
\hline & $\eta^{e \mu \mu}$ & $\eta^{e \mu \mu}$ & $\eta^{e \mu \mu}$ & & & & \\
\hline & $q_{\mu_{1}} \times q_{\mu_{2}}$ & & & & & $q_{e} \times \eta^{e}, q_{\mu} \times \eta^{\mu}$ & \\
\hline$\phi\left(e_{1}, e_{2}\right)$ & $\phi\left(\mu_{1}, \mu_{2}\right)$ & $\phi\left(\mu_{1}, \mu_{2}\right)$ & $\phi\left(\mu_{1}, \mu_{2}\right)$ & & & & \\
\hline$\phi(e e, \mu)$ & $\phi(\mu \mu, e)$ & $\phi(\mu \mu, e)$ & $\phi(\mu \mu, e)$ & & & & \\
\hline $\min \left\{\mathcal{R}\left(\ell, \ell^{\prime}\right)\right\}$ & $\min \left\{\mathcal{R}\left(\ell, \ell^{\prime}\right)\right\}$ & $\min \left\{\mathcal{R}\left(\ell, \ell^{\prime}\right)\right\}$ & $\min \left\{\mathcal{R}\left(\ell, \ell^{\prime}\right)\right\}$ & & & $\mathcal{R}(e, \mu)$ & \\
\hline \multirow[t]{2}{*}{$\operatorname{mid}\left\{\mathcal{R}\left(\ell, \ell^{\prime}\right)\right\}$} & & & $\operatorname{mid}\left\{\mathcal{R}\left(\ell, \ell^{\prime}\right)\right\}$ & & & & \\
\hline & & & $\max \left\{\mathcal{R}\left(\ell, \ell^{\prime}\right)\right\}$ & & & & \\
\hline$M(e e)$ & $M(\mu \mu)$ & $M(\mu \mu)$ & $M(\mu \mu)$ & $M(\tau \tau), M\left(\mu \tau_{1}\right)$ & $M(\tau \tau)$ & $M(e \mu)$ & \\
\hline$M\left(e e \mu \mathbb{E}_{T}\right)$ & $M(e \mu \mu)$ & & $M(e \mu \mu)$ & $M(\tau \tau \mu)$ & & & \\
\hline \multirow[t]{3}{*}{$\min \left\{M_{T}\left(e_{i}\right)\right\}$} & $\min \left\{M_{T}\left(\mu_{i}\right)\right\}$ & & & $M_{T}(\mu)$ & $M_{T}(\mu)$ & $\min \left\{M_{T}(e), M_{T}(\mu)\right\}$ & \\
\hline & $\max \left\{M_{T 2}\left(\ell \ell^{\prime} \mathscr{E}_{T}\right)\right\}$ & $\max \left\{M_{T 2}\left(\ell \ell^{\prime} \not \mathscr{E}_{T}\right)\right\}$ & & & & & \\
\hline & $\operatorname{mid}\left\{M_{T 2}\left(\ell \ell^{\prime} \mathscr{E}_{T}\right)\right\}$ & & & & & & \\
\hline \multirow[t]{9}{*}{$\mathbb{E}_{T}, \mathcal{S}\left(\not \mathbb{E}_{T}\right)$} & $\not \mathbb{E}_{T}, \mathcal{S}\left(\not \mathbb{E}_{T}\right)$ & $\not \mathbb{E}_{T}, \mathcal{S}\left(\not \mathbb{E}_{T}\right)$ & & $\not \mathscr{E}_{T}$ & $\not E_{T}$ & $\mathscr{E}_{T}$ & \\
\hline & & $M_{T}\left(e, \mathbb{E}_{T}\right)$ & & & & & \\
\hline & $\sum p_{T}^{\ell}+\not_{T}$ & $\sum p_{T}^{\ell}+\not E_{T}$ & $\sum p_{T}^{\ell}+\mathscr{E}_{T}$ & & & & \\
\hline & & $\min \left\{\phi\left(\mu_{i}, \mathbb{E}_{T}\right)\right\}$ & & & & $\max \left\{\left(\phi\left(\ell, \mathbb{E}_{T}\right)\right\}\right.$ & \\
\hline & & $N_{\text {jet }}$ & $N_{\text {jet }}$ & & & $N_{\text {jet }}$ & \\
\hline & $p_{T}^{\mathrm{jet}}$ & $p_{T}^{\mathrm{jet}}$ & $p_{T}^{\mathrm{jet}}$ & & & & $p_{T}^{\mathrm{jet}}$ \\
\hline & & & $\eta^{\text {jet }}$ & & & & \\
\hline & & $\max \left\{\phi\left(\right.\right.$ jet $\left.\left._{i}, \not \mathbb{E}_{T}\right)\right\}$ & & & & & $\phi\left(\right.$ jet, $\left.\mathscr{E}_{T}\right)$ \\
\hline & & & $f_{c p}$ & & & & \\
\hline
\end{tabular}

$M_{T}(\mu)>40 \mathrm{GeV}$ and $p_{T}^{\tau_{1}}>20 \mathrm{GeV}$. The normalization factors that are applied to the simulation are determined from the ratio of the event yields in the $W+$ jets enriched region for data and simulation. They are determined separately for each type of $\tau_{h}$ candidate, and for same-charge and opposite-charge $\tau_{h}$ pairs.

To normalize the simulated $W+$ jets background in the $e^{ \pm} \mu^{ \pm}$final state, we select a data sample enriched in $W+$ jets events by requiring $\mathbb{E}_{T}>20 \mathrm{GeV}$, an inverted electron likelihood criterion $\mathcal{L}_{e}<0.7$, and $\min \left\{M_{T}(e), M_{T}(\mu)\right\}>$ $20 \mathrm{GeV}$. This data sample is used to derive separate normalization factors for jet multiplicities $N_{\text {jet }}=0,1$, and $\geq 2$ that are applied to the simulated $W+$ jets background samples.
Multijet background is highly suppressed in final states with three high- $p_{T}$ isolated leptons. It is found to be negligible for the $e \mu \mu, e e \mu$, and $\mu \tau_{h} \tau_{h}$ final states using methods based on data. The multijet background in the $e^{ \pm} \mu^{ \pm}$channel is determined using an $e \mu$ multijet sample that contains events with one electron, selected with relaxed requirements on the likelihoods and neural networks compared to the standard electron identification, and with one muon, selected without applying isolation requirements. These electrons and muons are denoted as fake leptons. This sample has the same kinematic selection applied as the signal sample, but has no overlap with it due to the different lepton identification requirements. 
To obtain the correct normalization of this sample, we calculate separate fake rates for electrons and muons, given by the ratio of the number of events with a standard lepton to the number of events with a fake lepton. This fake rate is calculated separately for electrons and muons as a function of their $p_{T}$ in two inclusive samples. One sample is selected by requiring an electron in each event applying only relaxed identification requirements and the second sample by requiring a muon in each event but without applying muon isolation requirements. An additional requirement of $\not ̈_{T}<15 \mathrm{GeV}$ is applied for both samples, which ensures that they are enriched in multijet events. There is no significant overlap of the inclusive samples used to calculate the fake rate with the multijet $e \mu$ sample. The shape and normalization of the multijet contribution are then obtained by applying the product of the $p_{T}$-dependent fake rates for electrons and muons to the multijet $e \mu$ sample.

Photons reconstructed as electrons contribute to the background in the $e \mu \mu$ final state. This background is modeled using $\gamma \mu \mu$ data events, selected in the same way as the $e \mu \mu$ sample, except that the track matching criteria for electrons is reversed to reconstruct the photon. We then apply a normalization factor that describes the rate at which photons are misidentified as electrons. To calculate this normalization factor, we select events with two muons, a photon or electron (labeled $e / \gamma$ ), and $\not_{T}<$ $20 \mathrm{GeV}$. The invariant mass of the two muons and the $e / \gamma$ in the sample used to calculate the normalization factor has to be in the range between 75 and $105 \mathrm{GeV}$. This sample is enriched in events with final state radiation from $Z / \gamma^{*} \rightarrow \mu^{+} \mu^{-}$decays. The normalization factor is calculated as the ratio of the number of events with electrons to photons in this sample.

\section{MULTIVARIATE DISCRIMINANTS}

Boosted decision trees (BDTs), as implemented in the TMVA package [41], are used to discriminate between signal and background. The BDTs are trained for each mass point separately in the range $100 \leq M_{H} \leq$ $200 \mathrm{GeV}$, in steps of $5 \mathrm{GeV}$. For each background or signal process, the event samples are split into subsamples for training the BDTs. The BDTs are then applied to the subsamples not used in the training to derive limits on the Higgs boson production cross section. The only exception is the $Z \gamma$ background in the $e \mu \mu$ final state, where both simulation and data are used. We train the BDT with a $Z \gamma$ MC sample and then apply the BDT to the kinematic distributions estimated using the $Z \gamma$ data sample to obtain the BDT distribution used in the limit setting. This procedure reduces fluctuations in the training caused by the small number of data events.

The BDTs exploit kinematic differences between Higgs boson production for a given $M_{H}$ and background. The variables used as inputs to the BDTs are given in Table II.
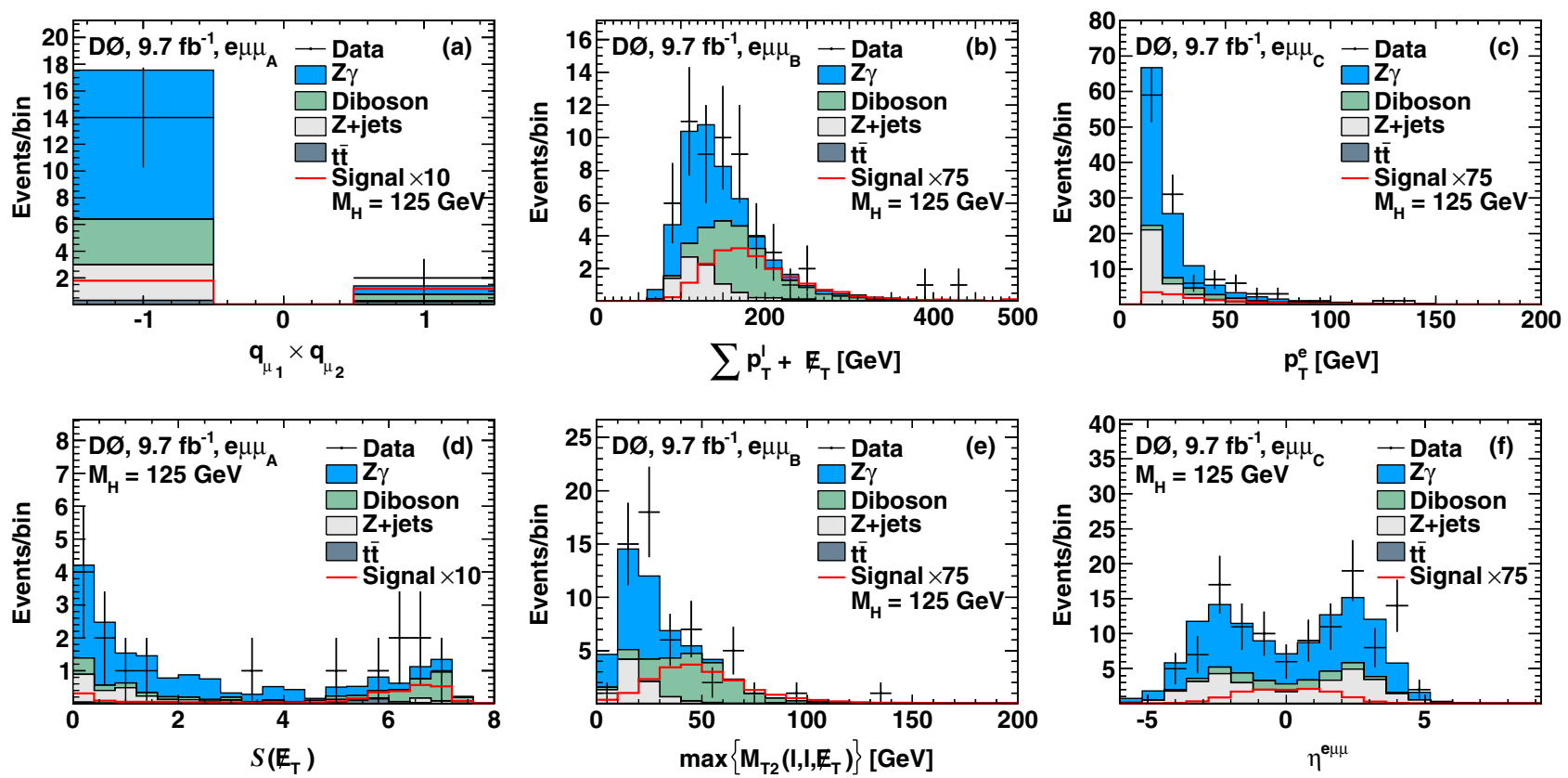

FIG. 1 (color online). Distributions of (a) the product of the muon charges, $q_{\mu_{1}} \times q_{\mu_{2}}$, (b) the scalar sum of transverse momenta and $\mathbb{E}_{T}, \sum p_{T}^{\ell}+\mathbb{E}_{T}$, (c) the transverse momentum of the electron, $p_{T}^{e}$, (d) the significance $\mathcal{S}\left(\mathbb{E}_{T}\right)$, (e) the maximal $M_{T 2}$ for all lepton pairings, $\max \left\{M_{T 2}\left(\ell \ell^{\prime} \mathscr{E}_{T}\right)\right\}$, and (f) the pseudorapidity of the trilepton system, $\eta^{e \mu \mu}$. The distributions are shown after the event selection. The $e \mu \mu_{\mathrm{A}, \mathrm{B}, \mathrm{C}}$ samples are shown in the left, middle, and right columns, respectively. The data are compared to the sum of the expected background and to simulations of a Higgs boson signal for $M_{H}=125 \mathrm{GeV}$, multiplied by a factor of 10 for the $e \mu \mu_{\mathrm{A}}$ channel and 75 for the other channels. 

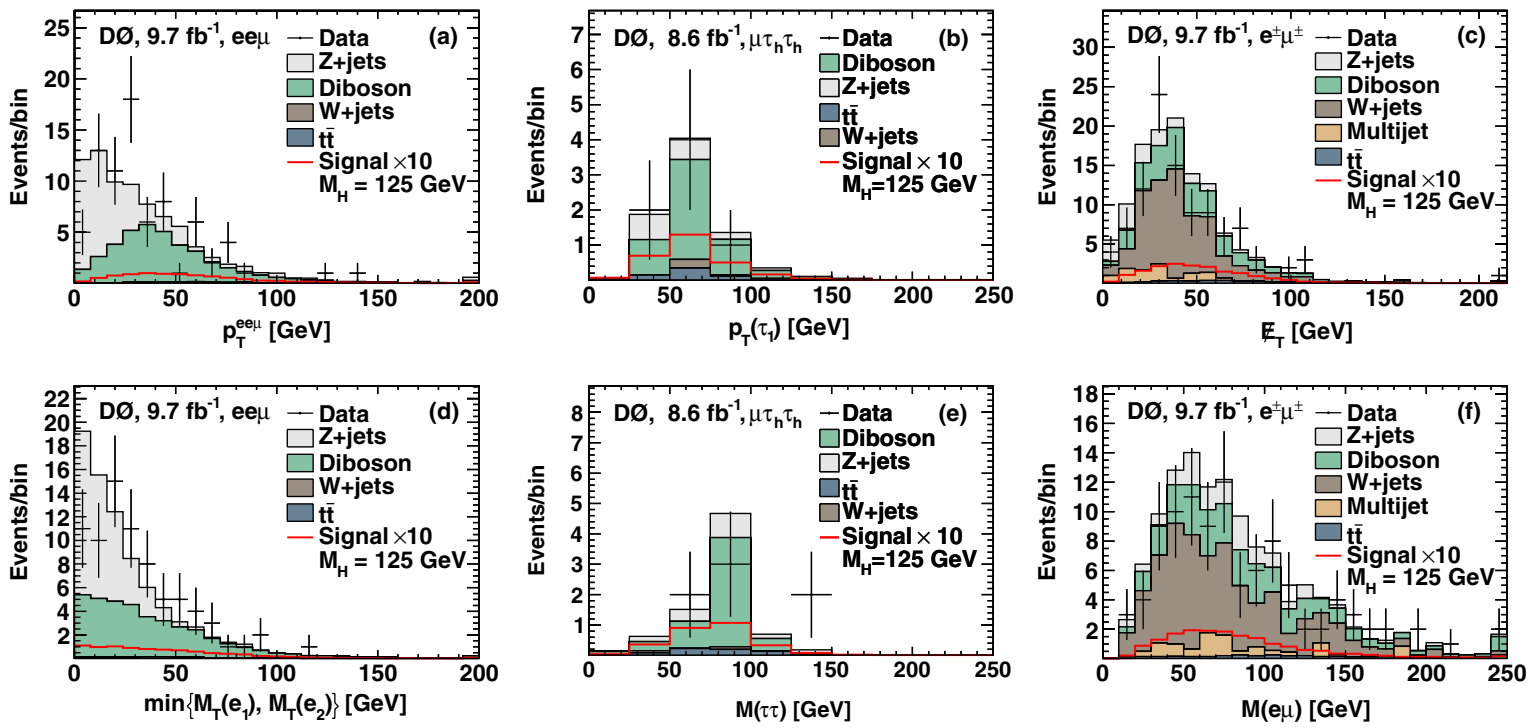

FIG. 2 (color online). Distributions of (a) trilepton transverse momentum, $p_{T}^{e e \mu}$, and (d) the minimal transverse mass, $\min \left\{M_{T}\left(e_{1}\right), M_{T}\left(e_{2}\right)\right\}$, for the $e e \mu$ samples, (b) the transverse momentum of the leading $\tau_{h}$ candidate, $p_{T}^{\tau_{1}}$, and (e) the ditau invariant mass, $M\left(\tau_{1}, \tau_{2}\right)$, for the $\mu \tau_{h} \tau_{h}$ samples, and (c) missing transverse energy, $\mathbb{E}_{T}$, and (f) the invariant mass of the electron and muon, $M_{e \mu}$, for the $e^{ \pm} \mu^{ \pm}$samples. The distributions are shown after the event selection. In addition, the final selection step requiring the output of the first BDT to be $>0.3$ and $\min \left\{M_{T}(e), M_{T}(\mu)\right\}>7 \mathrm{GeV}$ has also been applied to the $e^{ \pm} \mu^{ \pm}$sample. The data are compared to the sum of the expected background and to simulations of a Higgs boson signal for $M_{H}=125 \mathrm{GeV}$, multiplied by a factor of 10 .
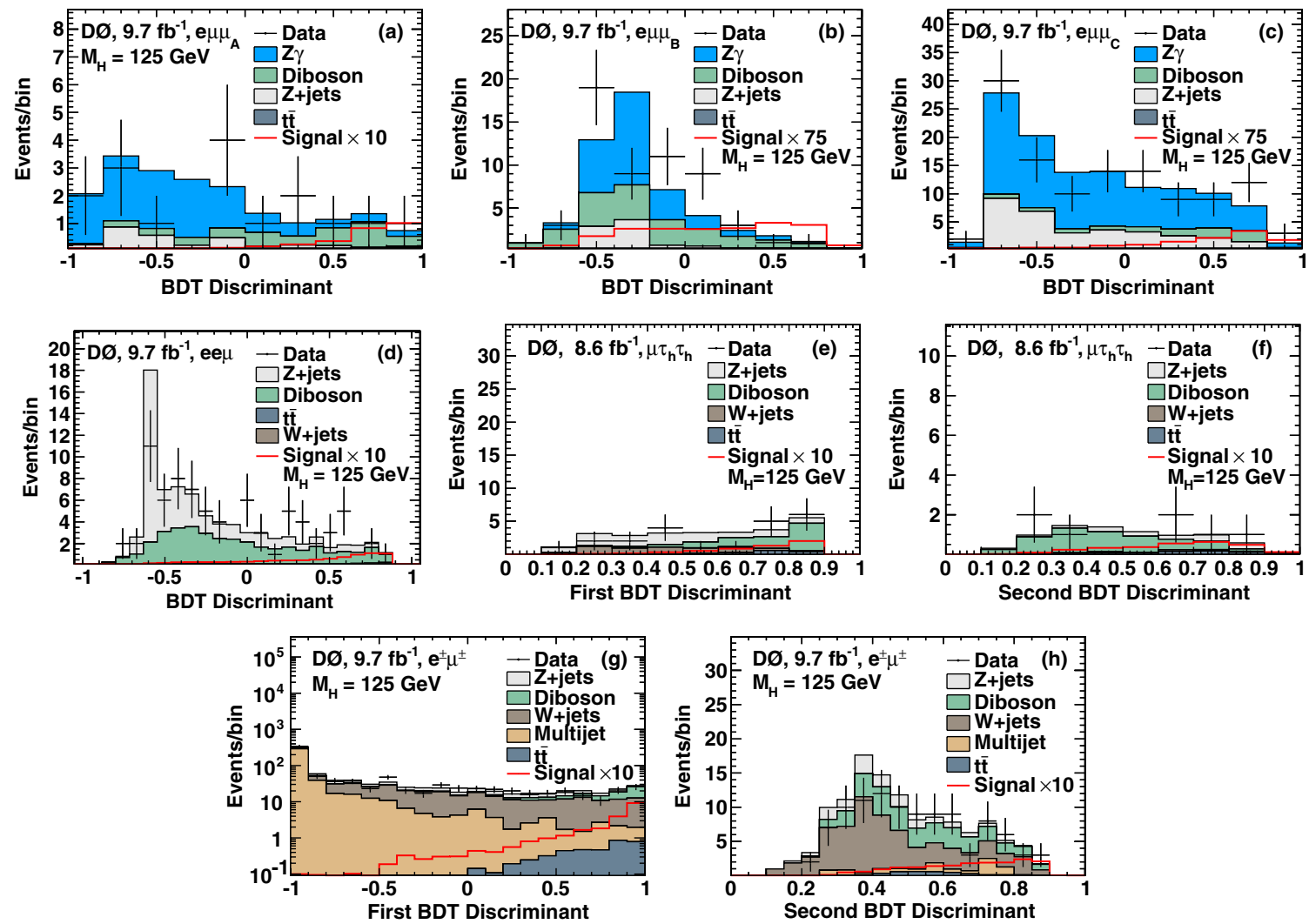

FIG. 3 (color online). Distribution of the BDT outputs for the (a) $e \mu \mu_{\mathrm{A}}$, (b) $e \mu \mu_{\mathrm{B}}$, (c) $e \mu \mu_{\mathrm{C}}$, and (d) $e e \mu$ channels and distributions of the outputs of the (e,g) first and (f,h) second BDT in the $\mu \tau_{h} \tau_{h}$ and $e^{ \pm} \mu^{ \pm}$channels, respectively. The data are compared to the sum of the expected background and to simulations of a Higgs boson signal for $M_{H}=125 \mathrm{GeV}$, multiplied by factors of 75 for the $e \mu \mu_{\mathrm{B}}$ and $e \mu \mu_{\mathrm{C}}$ channels and 10 for the other channels. 
They are based on the transverse momenta of the leptons and jets, the $\not{E}_{T}$, angular variables, charge correlations between leptons, and on the invariant masses of the pairings of leptons, jets, and the $\mathbb{E}_{T}$. Jet variables are used to calculate the discriminants in the $e \mu \mu$ and $e^{ \pm} \mu^{ \pm}$channels only. The variable $M_{T 2}$ is an extension of the transverse mass $M_{T}$ to final states with two visible and two invisible particles $[42,43]$. Some of the variables use constraints given by the $Z$ boson mass. On average, the opening angles between leptons in $H \rightarrow W W$ decays are smaller than for background and their direction is opposite to the direction of the $\not E_{T}$ because of spin correlations in the decay of a scalar Higgs boson. Other variables, such as the likelihood $\mathcal{L}_{e}$, reject events with misidentified leptons. Distributions of some of the input variables used for BDT training are shown in Figs. 1 and 2.

A single BDT for each mass point is used to discriminate between signal and all background processes for the $e e \mu$ sample and for each of the three $e \mu \mu$ subsamples. The output distributions of the BDTs [shown in Fig. 3(a)-3(d) for data, signal with $M_{H}=125 \mathrm{GeV}$, and for expected background] are used to discriminate between signal and background.

Two BDTs are trained for the $\mu \tau_{h} \tau_{h}$ sample, where the first BDT discriminates between signal and all background sources except diboson production and the second BDT between signal and the dominant diboson background. Events that pass a selection requirement on the first BDT discriminant of $>0.680-0.788$, determined separately for each $M_{H}$ value to optimize the discrimination between

TABLE III. Numbers of events in data, predicted background, and expected signal for $M_{H}=125 \mathrm{GeV}$ for the $e^{ \pm} \mu^{ \pm}$channel. The numbers are given after the initial event selection and after the final selection, which also requires the first BDT output to be $>0.3$ and $\min \left\{M_{T}(e), M_{T}(\mu)\right\}>7 \mathrm{GeV}$. The numbers of events are given with their total (statistical and systematic) uncertainties.

\begin{tabular}{lcc}
\hline \hline & Initial Selection & Final Selection \\
\hline Signal & & \\
$W H$ & 1.93 & 1.51 \\
$Z H$ & 0.32 & 0.23 \\
$g g \rightarrow H \rightarrow Z Z$ & $<0.01$ & $<0.01$ \\
Signal Sum & 2.25 & 1.74 \\
Background & & \\
$Z \rightarrow e^{+} e^{-}$ & $15.9 \pm 2.4$ & $2.7 \pm 0.4$ \\
$Z \rightarrow \mu^{+} \mu^{-}$ & $58.5 \pm 15.2$ & $10.6 \pm 2.8$ \\
$Z \rightarrow \tau^{+} \tau^{-}$ & $22.0 \pm 6.8$ & $1.8 \pm 0.6$ \\
$Z \gamma$ & $<0.1$ & $<0.1$ \\
Diboson & $36.2 \pm 3.6$ & $31.6 \pm 3.2$ \\
$t \bar{t}$ & $4.1 \pm 2.1$ & $3.4 \pm 1.7$ \\
$W+$ jets & $238.3 \pm 19.0$ & $62.4 \pm 5.0$ \\
Multijet & $434.5 \pm 87.0$ & $9.1 \pm 1.8$ \\
Background & & \\
Sum & $809 \pm 93$ & $122 \pm 7$ \\
Data & 822 & 102 \\
\hline \hline
\end{tabular}

signal and background, are used as input to the second BDT. The output distributions of the first BDT with $M_{H}=$ $125 \mathrm{GeV}$ are shown in Fig. 3(e) and for the second BDT in Fig. 3(f), using all events where the first BDT output is $>0.744$. The output of the first BDT is used as the discriminant in the limit setting for all events that fail the requirement on the first BDT output and the output of the second BDT for all remaining events.

The output distribution for the first BDT used for the $e^{ \pm} \mu^{ \pm}$channel is shown in Fig. 3(g). It discriminates mainly between signal and $W+$ jets as well as multijet production. After requiring the output of the first BDT to be $>0.3$ and $\min \left\{M_{T}(e), M_{T}(\mu)\right\}>7 \mathrm{GeV}$ in a final selection step, the number of expected background events at $M_{H}=125 \mathrm{GeV}$ is reduced from $809 \pm 93$ to $122 \pm 7$, while the expected number of signal events is only reduced by $23 \%$ (see Table III). A second BDT is trained to discriminate between signal and the remaining background sources, which are mainly from diboson, $W+$ jets, and $Z+$ jets production, in the remaining events. The output of the second BDT for this sample, shown in Fig. 3(h) for $M_{H}=125 \mathrm{GeV}$, is used as a discriminant in the Higgs boson searches.

\section{SYSTEMATIC UNCERTAINTIES}

Systematic uncertainties on both background and signal, including their correlations, are taken into account as uncertainties on the normalizations and on the shape of differential distributions. Table IV gives an overview of the sources of systematic uncertainties.

The theoretical uncertainty on signal production cross sections is $7.6 \%$ for gluon-gluon fusion and $6.2 \%$ for associated $V H$ production. Uncertainties on the background include the theoretical uncertainty on the cross sections for $Z / \gamma^{*} \rightarrow \ell^{+} \ell^{-}, W+$ jets, $t \bar{t}$, and diboson production, which vary between $5 \%$ and $7 \%$. The theoretical uncertainty on the $W+$ jets cross section is applied for the $e e \mu$ and $e \mu \mu$ channels, where this background is normalized using the theoretical prediction. The uncertainty from the normalization of the $W+$ jets background in the $\mu \tau_{h} \tau_{h}$ and $e^{ \pm} \mu^{ \pm}$channels is 5\%-6\%. Additional shapedependent uncertainties are applied in the $e^{ \pm} \mu^{ \pm}$channel to account for uncertainties in the description of the $p_{T}$ spectra of $W$ and $Z$ bosons as well as for initial and final state radiation in $W+$ jets events. The uncertainties on the $Z$ boson $p_{T}$ spectrum are applied as shape uncertainty in the $e e \mu$ and $e \mu \mu$ channels, whereas the uncertainty due to the $W$ boson $p_{T}$ spectrum is found to be negligible. The systematic uncertainty of $7.3 \%$ on the normalization of the $Z \gamma$ background in the $e \mu \mu$ final state is dominated by the statistical uncertainty on the normalization factor.

The shape-dependent uncertainty on charge misidentification for the $e \mu \mu$ channel is determined by comparing the ratios of same-charge and opposite-charge $\mu \mu$ events in data and MC. In the $e^{ \pm} \mu^{ \pm}$channel, the normalization 
TABLE IV. Sources of systematic uncertainties that affect either the shape of the differential distributions or the normalization of the expected signal and background for each final state.

\begin{tabular}{|c|c|c|c|c|c|}
\hline Source & Range & $e e \mu$ & $e \mu \mu$ & $\mu \tau_{h} \tau_{h}$ & $e^{ \pm} \mu^{ \pm}$ \\
\hline \multicolumn{6}{|l|}{ Signal } \\
\hline Higgs boson cross section $(g g \rightarrow H)$ & $7.6 \%$ & \multicolumn{4}{|c|}{ normalization } \\
\hline Higgs boson cross section $(\mathrm{VH})$ & $6.2 \%$ & \multicolumn{4}{|c|}{ normalization } \\
\hline \multicolumn{6}{|l|}{ Background } \\
\hline$Z / \gamma^{*} \rightarrow \ell^{+} \ell^{-}$cross section & $6 \%$ & norm & norm & norm & $\mathrm{n} / \mathrm{a}$ \\
\hline$W+$ jets & $5 \%-7 \%$ & \multicolumn{4}{|c|}{ normalization } \\
\hline$t \bar{t}$ cross section & $6 \%-10 \%$ & \multicolumn{4}{|c|}{ normalization } \\
\hline Diboson cross section & $6 \%-7 \%$ & \multicolumn{4}{|c|}{ normalization } \\
\hline$W+$ jets (initial/final state radiation) & & $\mathrm{n} / \mathrm{a}$ & $\mathrm{n} / \mathrm{a}$ & $\mathrm{n} / \mathrm{a}$ & shape \\
\hline$p_{T}$ spectrum of $W$ bosons & & $\mathrm{n} / \mathrm{a}$ & $\mathrm{n} / \mathrm{a}$ & $\mathrm{n} / \mathrm{a}$ & shape \\
\hline$p_{T}$ spectrum of $Z$ bosons & & shape & shape & $\mathrm{n} / \mathrm{a}$ & shape \\
\hline$Z / \gamma$ normalization & $7.3 \%$ & $\mathrm{n} / \mathrm{a}$ & norm & $\mathrm{n} / \mathrm{a}$ & $\mathrm{n} / \mathrm{a}$ \\
\hline Charge misidentification & & $\mathrm{n} / \mathrm{a}$ & shape & $\mathrm{n} / \mathrm{a}$ & norm \\
\hline Multijet background & $20 \%$ & $\mathrm{n} / \mathrm{a}$ & $\mathrm{n} / \mathrm{a}$ & $\mathrm{n} / \mathrm{a}$ & norm \\
\hline Signal and Background & & & & & \\
\hline Integrated luminosity & $6.1 \%$ & \multicolumn{4}{|c|}{ normalization } \\
\hline Parton distributions & $2.5 \%$ & norm & norm & $\mathrm{n} / \mathrm{a}$ & $\mathrm{n} / \mathrm{a}$ \\
\hline Inclusive trigger method & $1.5 \%-5 \%$ & \multicolumn{4}{|c|}{ normalization } \\
\hline$Z+$ jets normalization & $4 \%$ & $\mathrm{n} / \mathrm{a}$ & $\mathrm{n} / \mathrm{a}$ & $\mathrm{n} / \mathrm{a}$ & norm \\
\hline Lepton-in-jet misidentification & $30 \%$ & norm & norm & $\mathrm{n} / \mathrm{a}$ & $\mathrm{n} / \mathrm{a}$ \\
\hline Electron identification & $2.5 \%$ & norm & norm & $\mathrm{n} / \mathrm{a}$ & $\mathrm{n} / \mathrm{a}$ \\
\hline Muon identification & $2.9 \%-4 \%$ & norm & norm & norm & $\mathrm{n} / \mathrm{a}$ \\
\hline$\tau_{h}$ identification & $4 \%-7 \%$ & $\mathrm{n} / \mathrm{a}$ & $\mathrm{n} / \mathrm{a}$ & norm & $\mathrm{n} / \mathrm{a}$ \\
\hline Electron energy scale & & shape & shape & $\mathrm{n} / \mathrm{a}$ & $\mathrm{n} / \mathrm{a}$ \\
\hline Muon energy scale & & shape & shape & $\mathrm{n} / \mathrm{a}$ & $\mathrm{n} / \mathrm{a}$ \\
\hline Jet energy & & $\mathrm{n} / \mathrm{a}$ & shape & $\mathrm{n} / \mathrm{a}$ & norm/shape \\
\hline Jet reconstruction & & $\mathrm{n} / \mathrm{a}$ & shape & $\mathrm{n} / \mathrm{a}$ & norm/shape \\
\hline
\end{tabular}

TABLE V. Expected and observed 95\% C.L. upper limits on the SM Higgs boson production cross section relative to the SM expected value, for the $e e \mu, e \mu \mu, \mu \tau_{h} \tau_{h}$, and $e^{ \pm} \mu^{ \pm}$channels separately and combined.

\begin{tabular}{lrrrrrrrrrr}
\hline \hline & \multicolumn{2}{c}{$e e \mu$} & \multicolumn{2}{c}{$e \mu \mu$} & \multicolumn{2}{c}{$\mu \tau_{h} \tau_{h}$} & \multicolumn{2}{c}{$e^{ \pm} \mu^{ \pm}$} & \multicolumn{2}{c}{ Combined } \\
$M_{H}(\mathrm{GeV})$ & Exp & Obs & Exp & Obs & Exp & Obs & Exp & Obs & Exp & Obs \\
\hline 100 & 16.6 & 36.1 & 24.8 & 32.9 & 8.2 & 10.8 & 18.6 & 10.4 & 6.3 & 7.5 \\
105 & 17.4 & 36.1 & 23.5 & 24.0 & 9.3 & 11.4 & 19.3 & 12.3 & 6.7 & 7.2 \\
110 & 18.6 & 34.8 & 24.0 & 38.2 & 10.2 & 12.3 & 18.9 & 13.0 & 7.4 & 7.2 \\
115 & 17.7 & 34.1 & 22.3 & 27.1 & 11.3 & 13.6 & 17.8 & 12.9 & 7.1 & 10.9 \\
120 & 16.5 & 28.6 & 21.7 & 22.5 & 12.7 & 17.2 & 14.4 & 9.8 & 7.3 & 9.6 \\
125 & 14.1 & 19.9 & 17.0 & 22.3 & 13.0 & 19.4 & 11.8 & 8.8 & 6.3 & 8.4 \\
130 & 12.3 & 17.4 & 14.3 & 15.4 & 13.5 & 13.3 & 10.4 & 7.4 & 5.9 & 5.5 \\
135 & 11.0 & 16.0 & 13.1 & 12.4 & 14.6 & 17.6 & 8.4 & 6.2 & 5.1 & 4.9 \\
140 & 10.1 & 12.6 & 11.4 & 11.3 & 14.1 & 20.6 & 8.5 & 7.2 & 4.9 & 5.2 \\
145 & 9.4 & 11.2 & 11.2 & 11.4 & 14.2 & 22.3 & 7.7 & 6.4 & 4.6 & 5.1 \\
150 & 8.9 & 11.7 & 10.6 & 9.8 & 16.2 & 20.1 & 7.0 & 6.9 & 4.3 & 5.2 \\
155 & 9.3 & 11.5 & 10.8 & 9.0 & 15.4 & 17.6 & 7.3 & 6.2 & 4.4 & 4.5 \\
160 & 9.6 & 10.7 & 10.9 & 9.3 & 15.4 & 22.8 & 6.9 & 5.9 & 4.2 & 4.4 \\
165 & 9.6 & 9.3 & 10.3 & 8.5 & 16.1 & 23.9 & 6.6 & 6.3 & 4.1 & 4.6 \\
170 & 11.0 & 10.7 & 11.0 & 12.3 & 16.0 & 16.2 & 7.5 & 7.2 & 4.5 & 4.7 \\
175 & 11.9 & 10.6 & 12.7 & 22.4 & 17.4 & 34.3 & 7.9 & 8.3 & 5.0 & 7.7 \\
180 & 12.9 & 11.3 & 13.5 & 16.7 & 21.1 & 40.7 & 8.5 & 10.4 & 5.6 & 7.3 \\
185 & 13.6 & 13.0 & 14.1 & 19.8 & 20.1 & 26.2 & 9.9 & 11.3 & 6.0 & 10.6 \\
190 & 14.4 & 13.1 & 15.1 & 29.1 & 24.0 & 36.7 & 10.7 & 14.5 & 6.7 & 11.2 \\
195 & 15.5 & 13.8 & 17.1 & 25.6 & 25.9 & 37.8 & 12.8 & 17.3 & 7.7 & 12.7 \\
200 & 16.4 & 11.6 & 17.8 & 23.7 & 27.5 & 33.3 & 11.9 & 17.3 & 7.7 & 10.1 \\
\hline \hline
\end{tabular}



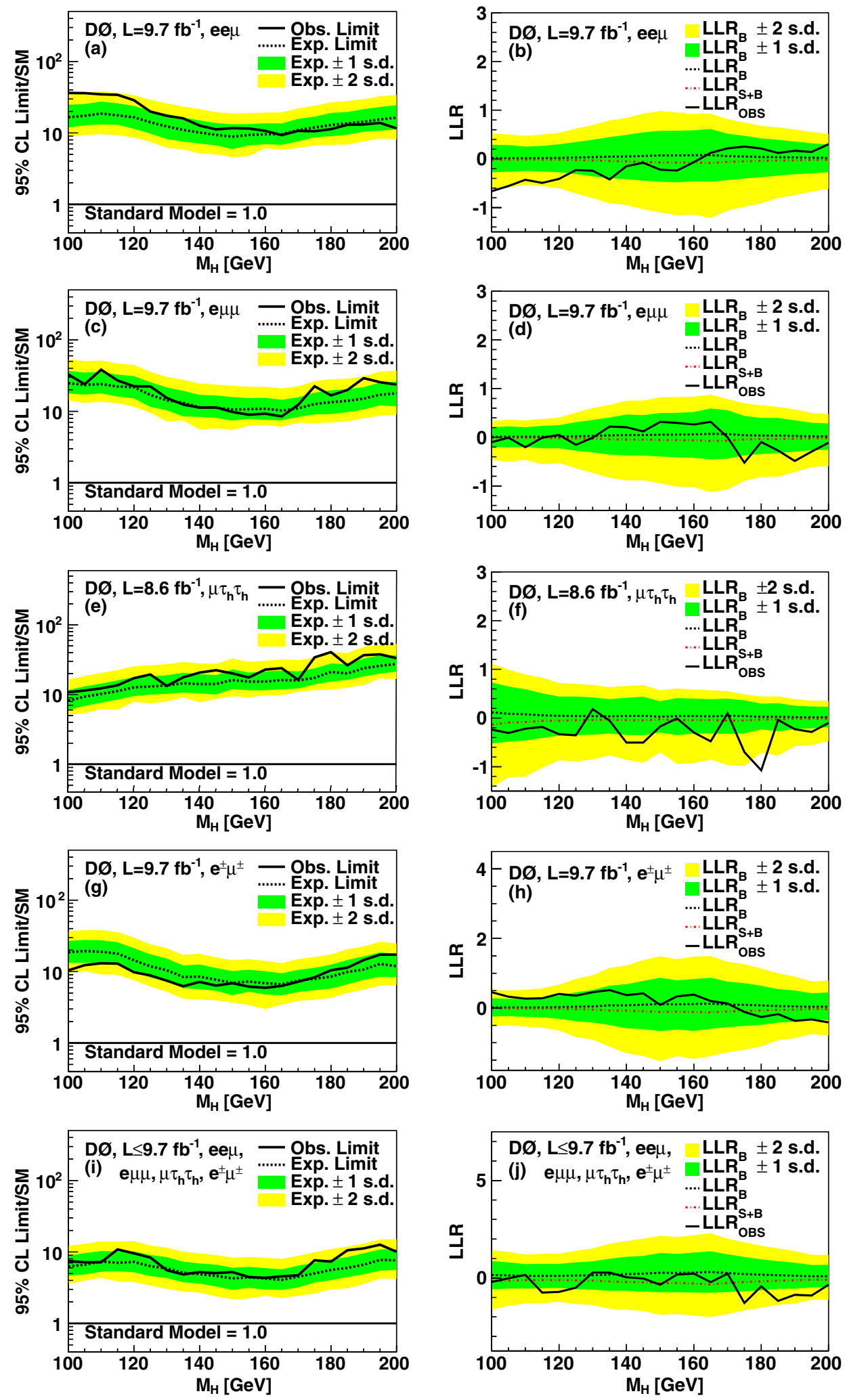

FIG. 4 (color online). Upper limit on the SM Higgs boson production cross section expressed as the ratio to the SM prediction (left column) and observed LLR (right column) as a function of $M_{H}$ for the (a,b) $e e \mu$, (c,d) $e \mu \mu$, (e,f) $\mu \tau_{h} \tau_{h}$, (g,h) $e^{ \pm} \mu^{ \pm}$channels, and (i,j) for all channels combined. The LLRs are shown for the background-only and the signal-plus-background hypotheses. The bands correspond to regions of \pm 1 and \pm 2 standard deviations (s.d.) around the median expected limit and around the expected median LLR for the background-only hypothesis, respectively. 
uncertainty on the background from charge misidentification is $16 \%$ for $Z+$ jets, $50 \%$ for $t \bar{t}$, and $7.5 \%$ for diboson background, assessed by studying the contribution of charge misidentification in the simulation. The systematic uncertainty on the normalization of the multijet background in the $e^{ \pm} \mu^{ \pm}$channel is estimated to be $20 \%$ by studying its dependence on jet multiplicity and lepton $p_{T}$.

The uncertainty on the measured integrated luminosity is $6.1 \%$ [44], affecting both signal and simulated background. The uncertainty on the acceptance from the uncertainty on the parton distribution functions is $2.5 \%$. Uncertainties on the normalization of the signal and the simulated background are studied by comparing distributions of data obtained using the inclusive trigger method with those obtained using only single-lepton triggers. The uncertainty of $1.5 \%-5 \%$ from using the inclusive trigger method is calculated from the statistical uncertainty of the data samples used to derive the trigger corrections.

An overall normalization uncertainty is applied to the $e^{ \pm} \mu^{ \pm}$sample, which is normalized to the $Z+$ jets cross section using opposite-charge $e \mu$ events. It is given by the uncertainty on the $Z+$ jets cross section of $4 \%$ and is therefore applied to all simulated signal and background samples, except the $Z+$ jets contribution itself.

The uncertainty on the probability that leptons originating from jets in $W+$ jets, $Z+$ jets or diboson events pass the selection in the $e e \mu$ and $e \mu \mu$ channels is estimated to be $30 \%$. Uncertainties on the lepton identification efficiencies are applied in the trilepton channels. They are 1.8\%$4 \%$ per muon and $2.5 \%$ per electron. The uncertainty on the identification efficiency for $\tau_{h}$ candidates, including the uncertainty on the neural network discriminant used to distinguish $\tau_{h}$ candidates from jets and the $\tau_{h}$ energy scale, is $4 \%-7 \%$ per $\tau_{h}$ candidate. The uncertainties on the $p_{T}$ resolutions and energy scale for electrons and muons, and the uncertainties from jet energy resolution and efficiencies, derived using the prescription given in Ref. [40], are found to have a negligible effect on the results.

\section{RESULTS ON THE SM HIGGS BOSON}

We determine limits on the SM Higgs boson production cross section using a modified frequentist approach [45] with the distributions of the final discriminants shown in Fig. 3. A log-likelihood ratio (LLR) test statistic is formed using the Poisson probabilities for estimated background yields, the signal acceptance, and the observed number of events for different Higgs boson mass hypotheses. The confidence levels are derived by integrating the LLR distribution in pseudo-experiments using both the signalplus-background $\left(\mathrm{CL}_{\mathrm{sb}}\right)$ and the background-only hypotheses $\left(\mathrm{CL}_{\mathrm{b}}\right)$. The excluded production cross section is taken to be the cross section for which the confidence level for signal, $\mathrm{CL}_{\mathrm{s}}=\mathrm{CL}_{\mathrm{sb}} / \mathrm{CL}_{\mathrm{b}}$, equals 0.05 . The limits on the cross section for the different final states are given in

TABLE VI. Expected and observed 95\% C.L. upper limits on the fermiophobic Higgs boson production cross section relative to the expected cross section in the fermiophobic model, for the $e e \mu, e \mu \mu, \mu \tau_{h} \tau_{h}$, and $e^{ \pm} \mu^{ \pm}$channels separately and combined.

\begin{tabular}{lrrrrrrrrrr}
\hline \hline & \multicolumn{2}{c}{$e e \mu$} & \multicolumn{2}{c}{$e \mu \mu$} & \multicolumn{2}{c}{$\mu \tau_{h} \tau_{h}$} & \multicolumn{2}{c}{$e^{ \pm} \mu^{ \pm}$} & \multicolumn{2}{c}{ Combined } \\
$M_{H}(\mathrm{GeV})$ & Exp & Obs & Exp & Obs & Exp & Obs & Exp & Obs & Exp & Obs \\
\hline 100 & 5.1 & 10.4 & 5.7 & 7.6 & 26 & 27 & 3.7 & 2.1 & 2.6 & 2.5 \\
105 & 4.4 & 9.5 & 5.1 & 5.0 & 17 & 16 & 3.7 & 2.4 & 2.3 & 2.5 \\
110 & 5.3 & 9.2 & 5.4 & 9.1 & 16 & 13 & 3.4 & 2.3 & 2.3 & 2.7 \\
115 & 4.6 & 8.6 & 4.9 & 6.2 & 15 & 15 & 3.8 & 2.7 & 2.3 & 2.7 \\
120 & 5.1 & 8.5 & 5.8 & 6.5 & 17 & 25 & 3.7 & 2.5 & 2.5 & 2.5 \\
125 & 5.1 & 7.0 & 5.8 & 7.7 & 17 & 18 & 3.7 & 2.8 & 2.5 & 2.5 \\
130 & 5.5 & 7.5 & 6.1 & 6.6 & 18 & 17 & 4.1 & 3.0 & 2.7 & 2.4 \\
135 & 6.0 & 8.3 & 6.9 & 6.1 & 17 & 20 & 4.1 & 3.1 & 2.8 & 2.6 \\
140 & 6.7 & 8.3 & 7.2 & 6.8 & 17 & 18 & 5.1 & 4.1 & 3.2 & 2.8 \\
145 & 7.2 & 8.3 & 8.1 & 8.3 & 16 & 20 & 5.3 & 4.5 & 3.4 & 3.3 \\
150 & 7.7 & 9.8 & 8.9 & 7.6 & 20 & 28 & 5.6 & 5.4 & 3.5 & 4.0 \\
155 & 8.4 & 10.7 & 9.7 & 7.9 & 21 & 23 & 6.5 & 5.4 & 4.0 & 3.8 \\
160 & 9.4 & 10.5 & 10.4 & 9.0 & 19 & 30 & 6.6 & 5.7 & 4.1 & 4.2 \\
165 & 9.4 & 9.3 & 10.0 & 8.3 & 22 & 32 & 6.6 & 6.1 & 4.2 & 4.0 \\
170 & 11.2 & 10.8 & 11.2 & 12.1 & 23 & 30 & 7.3 & 7.0 & 4.7 & 4.9 \\
175 & 12.1 & 10.6 & 12.7 & 21.9 & 25 & 29 & 7.9 & 8.2 & 5.1 & 6.5 \\
180 & 13.5 & 11.4 & 14.4 & 16.4 & 29 & 33 & 8.5 & 10.1 & 5.8 & 6.5 \\
185 & 14.8 & 13.7 & 15.3 & 20.6 & 31 & 45 & 10.0 & 11.4 & 6.4 & 8.5 \\
190 & 16.3 & 13.5 & 17.0 & 30.5 & 35 & 50 & 11.0 & 14.4 & 7.2 & 10.8 \\
195 & 17.8 & 15.5 & 19.7 & 29.1 & 40 & 62 & 12.9 & 17.3 & 8.2 & 13.0 \\
200 & 19.0 & 12.0 & 20.6 & 23.9 & 43 & 47 & 12.1 & 17.3 & 8.5 & 9.9 \\
\hline \hline
\end{tabular}



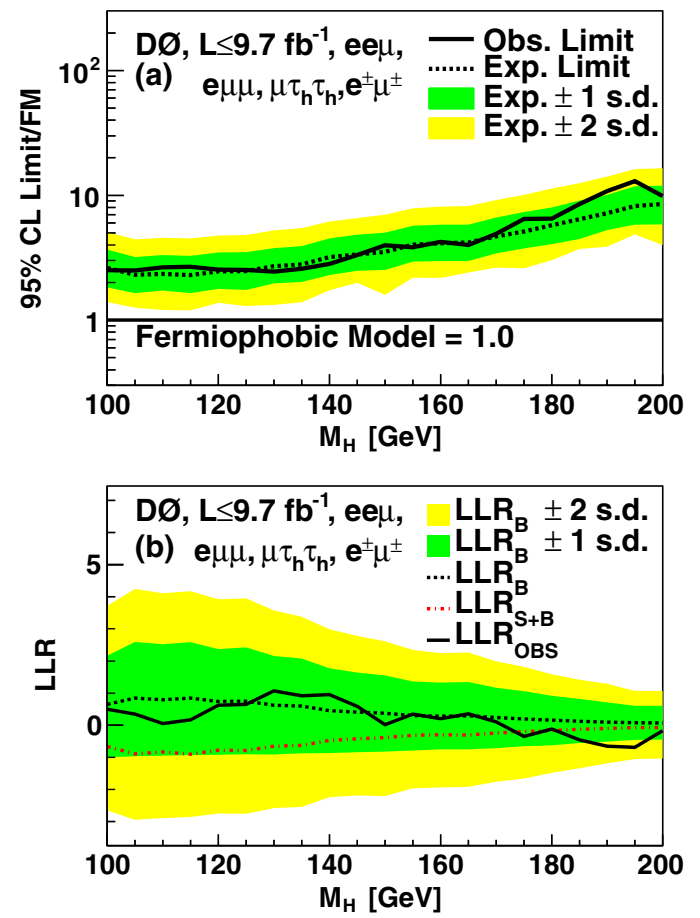

FIG. 5 (color online). (a) Upper limit on the fermiophobic Higgs boson production cross section expressed as a ratio to the prediction and (b) observed LLR as a function of $M_{H}$ for the combined channels. Also shown are the expected LLR distributions for the background-only hypothesis and for the signal + background hypothesis, with the bands indicating \pm 1 and \pm 2 s.d. fluctuations around the expected median LLR for the background-only hypothesis.

Table V. The individual channels have similar sensitivity, and the combined upper limits only vary within about a factor of 2 over the entire mass range of $100 \leq M_{H}<$ $200 \mathrm{GeV}$. At $M_{H}=125 \mathrm{GeV}$ the expected and observed upper limits on the cross section, expressed as a ratio relative to the predicted SM cross section, are 6.3 and 8.4, respectively. Figure 4 shows the limits on the cross section and the LLR distributions for each channel and for the combined result.

\section{RESULTS ON FERMIOPHOBIC HIGGS BOSONS}

In addition, we set limits in a fermiophobic Higgs boson model, where the Higgs boson is assumed to couple to $W$ and $Z$ bosons with SM strengths and the Higgs boson couplings to fermions are zero. The gluon-gluon fusion
Higgs production cross section is therefore small and is neglected. The outputs of the BDTs trained using a SM Higgs boson signal are used to set the limits with signal distributions where the gluon-gluon fusion processes and the $H \rightarrow \tau^{+} \tau^{-}$decays have been removed and only the $V H$ production mechanism is considered. The limits on the cross section, given as a ratio relative to the cross section in the fermiophobic model, are listed in Table VI. The $e e \mu$, $e \mu \mu$, and $e^{ \pm} \mu^{ \pm}$channels have similar sensitivity to a fermiophobic Higgs boson, whereas the $\mu \tau_{h} \tau_{h}$ channel is less sensitive because $H \rightarrow \tau^{+} \tau^{-}$decays do not contribute. The combined expected upper limits vary between 2.3 and 8.5 and the observed limits between 2.4 and 13.0, expressed as a ratio relative to the cross section in the fermiophobic model. Figure 5 shows the limits on the cross section for the production of a fermiophobic Higgs boson and the LLR distribution.

\section{CONCLUSION}

We have presented the first search for the SM Higgs boson in multilepton $e e \mu, e \mu \mu$, and $\mu \tau_{h} \tau_{h}$ final states and in like-charge $e^{ \pm} \mu^{ \pm}$final states using the $\mathrm{D} 0$ detector. The search is mainly sensitive to associated production of a $W$ or $Z$ boson with a Higgs boson, where the Higgs boson decays into $W^{+} W^{-}$and $Z Z$ pairs, thereby probing the $H V V$ coupling directly in production and decay. We set limits on the cross section for a SM Higgs boson in the range $100 \leq M_{H} \leq 200 \mathrm{GeV}$ with expected and observed upper limits of 6.3 and 8.4 at $M_{H}=125 \mathrm{GeV}$, expressed as the ratio relative to the predicted SM cross section. We also interpret the data in a fermiophobic Higgs boson model.

\section{ACKNOWLEDGMENTS}

We thank the staffs at Fermilab and collaborating institutions, and acknowledge support from the DOE and NSF (USA); CEA and CNRS/IN2P3 (France); MON, NRC KI and RFBR (Russia); CNPq, FAPERJ, FAPESP and FUNDUNESP (Brazil); DAE and DST (India); Colciencias (Colombia); CONACyT (Mexico); NRF (Korea); FOM (The Netherlands); STFC and the Royal Society (United Kingdom); MSMT and GACR (Czech Republic); BMBF and DFG (Germany); SFI (Ireland); The Swedish Research Council (Sweden); and CAS and CNSF (China).
[1] G. Aad et al. (ATLAS Collaboration), Phys. Lett. B 716, 1 (2012).

[2] S. Chatrchyan et al. (CMS Collaboration), Phys. Lett. B 716, 30 (2012).
[3] T. Aaltonen et al. (CDF and D0 Collaborations), Phys. Rev. Lett. 109, 071804 (2012); 109, 111802 (2012); V. M. Abazov et al. (D0 Collaboration), Phys. Rev. Lett. 109, 121802 (2012). 
[4] V. M. Abazov et al. (D0 Collaboration), this issue, Phys. Rev. D 88, 052006 (2013).

[5] V. M. Abazov et al. (D0 Collaboration), Phys. Rev. D 84, 092002 (2011).

[6] V. M. Abazov et al. (D0 Collaboration), this issue, Phys. Rev. D 88, 052005 (2013).

[7] J. Abdallah et al. (DELPHI Collaboration), Eur. Phys. J. C 35, 313 (2004).

[8] A. Heister et al. (ALEPH Collaboration), Phys. Lett. B 544, 16 (2002).

[9] M. Acciarri et al. (L3 Collaboration), Phys. Lett. B 489, 115 (2000).

[10] G. Abbiendi et al. (OPAL Collaboration), Phys. Lett. B 544, 44 (2002).

[11] V. M. Abazov et al. (D0 Collaboration), Phys. Rev. Lett. 107, 151801 (2011).

[12] T. Aaltonen et al. (CDF Collaboration), Phys. Rev. Lett. 103, 061803 (2009).

[13] S. Chatrchyan et al. (CMS Collaboration), J. High Energy Phys. 09 (2012) 111.

[14] V. M. Abazov et al. (D0 Collaboration), this issue, Phys. Rev. D 88, 052011 (2013).

[15] T. Aaltonen et al. (CDF and D0 Collaborations), this issue, Phys. Rev. D 88, 052014 (2013).

[16] V. M. Abazov et al. (D0 Collaboration), Nucl. Instrum. Methods Phys. Res., Sect. A 565, 463 (2006); M. Abolins et al., Nucl. Instrum. Methods Phys. Res., Sect. A 584, 75 (2008); R. Angstadt et al., Nucl. Instrum. Methods Phys. Res., Sect. A 622, 298 (2010).

[17] The pseudorapidity is given by $\eta=-\ln [\tan (\theta / 2)]$, where $\theta$ is the polar angle with respect to the proton beam direction. We define $\eta$ with respect to the $p \bar{p}$ interaction vertex and detector $\eta\left(\eta_{d}\right)$ with respect to the nominal center of the detector.

[18] V. M. Abazov et al. (D0 Collaboration), Nucl. Instrum. Methods Phys. Res., Sect. A 552, 372 (2005).

[19] M. L. Mangano, F. Piccinini, A. D. Polosa, M. Moretti, and R. Pittau, J. High Energy Phys. 07 (2003) 001; we use version 2.11 .

[20] T. Sjöstrand, S. Mrenna, and P. Skands, J. High Energy Phys. 05 (2006) 026; we use version 6.323.

[21] J. Pumplin, D. R. Stump, J. Huston, H.-L. Lai, P. Nadolsky, and W.-K. Tung, J. High Energy Phys. 07 (2002) 012; D. Stump, J. Huston, J. Pumplin, W.-K. Tung, H.-L. Lai, S. Kuhlmann, and J. F. Owens, J. High Energy Phys. 10 (2003) 046.
[22] Z. Wass, Nucl. Phys. B, Proc. Suppl. 98, 96 (2001); we use version 2.5.04.

[23] U. Langenfeld, S. Moch, and P. Uwer, Phys. Rev. D 80, 054009 (2009).

[24] J. M. Campbell and R. K. Ellis, Phys. Rev. D 60, 113006 (1999).

[25] T. Melia, P. Nason, R. Rontsch, and G. Zanderighi, J. High Energy Phys. 11 (2011) 078. This correction is only applied for the $е e \mu$ and $e \mu \mu$ channels.

[26] R. Hamberg, W. L. van Neerven, and T. Matsuura, Nucl. Phys. B359, 343 (1991); B644, 403(E) (2002).

[27] V. M. Abazov et al. (D0 Collaboration), Phys. Rev. Lett. 100, 102002 (2008).

[28] K. Melnikov and F. Petriello, Phys. Rev. D 74, 114017 (2006).

[29] J. Baglio and A. Djouadi, J. High Energy Phys. 10 (2010) 064.

[30] P. Bolzoni, F. Maltoni, S.-O. Moch, and M. Zaro, Phys. Rev. Lett. 105, 011801 (2010).

[31] D. de Florian and M. Grazzini, Phys. Lett. B 674, 291 (2009).

[32] A. D. Martin, W. J. Stirling, R. S. Thorne, and G. Watt, Eur. Phys. J. C 63, 189 (2009).

[33] G. Bozzi, S. Catani, D. de Florian, and M. Grazzini, Phys. Lett. B 564, 65 (2003); Nucl. Phys. B737, 73 (2006).

[34] A. Djouadi, J. Kalinowski, and M. Spira, Comput. Phys. Commun. 108, 56 (1998).

[35] R. Brun and F. Carminati, CERN Program Library Long Writeup W5013 (1993).

[36] V. M. Abazov et al. (D0 Collaboration), Phys. Rev. D 76, 012003 (2007).

[37] V. M. Abazov et al. (D0 Collaboration), Phys. Rev. D 71, 072004 (2005); 77, 039901(E) (2008).

[38] A. Schwartzman, Report No. FERMILAB-THESIS-200421.

[39] G. Blazey et al., arXiv:hep-ex/0005012.

[40] V. M. Abazov et al. (D0 Collaboration), Phys. Rev. D 85, 052006 (2012).

[41] H. Voss et al., Proc. Sci., ACAT2007 (2007) 040 [arXiv: physics/0703039]; we use version 4.1.0.

[42] C. Lester and D. Summers, Phys. Lett. B 463, 99 (1999).

[43] H. Cheng and Z. Han, J. High Energy Phys. 12 (2008) 063.

[44] T. Andeen et al., Report No. FERMILAB-TM-2365, 2007.

[45] T. Junk, Nucl. Instrum. Methods Phys. Res., Sect. A 434, 435 (1999); W. Fisher, Report No. FERMILAB-TM-2386E, 2006. 